\title{
Prevalent and Dynamic Binding of the Cell Cycle Checkpoint Kinase
} Rad53 to Gene Promoters

\author{
Yi-Jun Sheu, Risa Karakida Kawaguchi, Jesse Gillis and Bruce Stillman ${ }^{1}$ \\ Cold Spring Harbor Laboratory, 1 Bungtown Road, Cold Spring Harbor, NY 11724, USA \\ ${ }^{1}$ Correspondence: Bruce Stillman stillman@cshl.edu
}

Key words: Origins of DNA replication, Transcription start sites, Gene promoters, Checkpoint Kinase, Rad53, Mrc1, DNA damage response, Stress Response

\section{Abstract}

Replication of the genome must be coordinated with gene transcription and cellular metabolism.

These processes are controlled in part by the Rad53 (CHEK2 in mammals) checkpoint kinase and the Mrc1 replisome component, especially following replication stress in the presence of limiting deoxyribonucleotides. We examined cell cycle regulated, genome-wide binding of Rad53 to chromatin. The kinase bound to sites of active DNA replication initiation and fork progression, but unexpectedly to the promoters of numerous genes ( $>20 \%$ of all genes) involved in many cellular functions. At some genes, Rad53 promoter binding correlated with changes in gene expression. Rad53 promoter binding to certain genes is influenced by sequence-specific transcription factors and less by checkpoint signaling. In checkpoint mutants, untimely activation of late-replicating origins reduces the transcription of nearby genes, with concomitant localization of Rad53 to their gene bodies. We suggest that the Rad53 checkpoint kinase coordinates genome-wide replication and transcription under stress conditions. 


\section{Introduction}

Eukaryotic cells initiate DNA synthesis in a temporally controlled manner from multiple replication origins to ensure efficient duplication of the genome (Bell and Labib, 2016; Renard-Guillet et al., 2014). During the course of replication, replisomes have to deal with both endogenous and exogenous stresses that can cause stalling of replication forks. The same DNA template is also transcribed, potentially creating conflicts between replication and transcription that can lead to detrimental effects on genome stability and cell viability (Hamperl and Cimprich, 2016).

To maintain genome stability during S-phase, the budding yeast $S$. cerevisiae activates a DNA replication checkpoint (DRC) in response to replication stress via the sensor kinase Mec1 (the mammalian ATM/ATR), the replication fork protein Mrc1 (Claspin in mammals) and other fork proteins (Lanz et al., 2019; Osborn and Elledge, 2003; Pardo et al., 2017; Paulovich and Hartwell, 1995; Saldivar et al., 2017). A second DNA damage checkpoint (DDC) mediated by Rad9 (TP53BP1 in mammals) responds to double strand DNA breaks. Both branches converge on the effector kinase Rad53 (CHEK2 in mammals) which triggers a wide range of downstream events, including stopping cell cycle progression, preventing late origin firing, activating the DNA repair and elevating synthesis of deoxyribonucleoside triphosphates (dNTP). The signaling also promotes widespread changes in gene expression (Jaehnig et al., 2013; Pardo et al., 2017).

Unlike most of the checkpoint genes, both Mec1 and Rad53 kinases are essential for cell viability in unperturbed cells that can be partly explained by their role in regulating dNTP pools (Desany et al., 1998; Forey et al., 2020; Zhao et al., 2000). However, it is important to note that kinase null mutants are extremely sick and sensitive to various type of exogenous stress. Under the bypass conditions in cells without Sml1, the inhibitor of ribonucleotide reductase (RNR), cells lacking Rad53 exhibit a more severe defect than cells lacking Mec1, implying that Rad53 has activities beyond checkpoint signaling. Consistent with this suggestion, the kinase deficient mutant $\mathrm{rad} 53^{\mathrm{K} 227 \mathrm{~A}}$ lacks checkpoint function but retains growth-associated activity (Gunjan and Verreault, 2003; Hoch et al., 2013; Holzen and Sclafani, 2010; Pellicioli et al., 1999).

Rad53 is central to the transcriptional response to DNA damage, including the Dun1 protein kinase acting downstream of Rad53 to phosphorylate and inactivate the transcriptional repressor Rfx1/Crt1 and thereby up-regulate target genes (Huang et al., 1998), such as $R N R 2, R N R 3$, and $R N R 4$, all encoding subunits of RNR. However, the induced expression of $R N R 1$, which encodes the major isoform of the RNR large subunit, is not controlled by the Rfx1 repressor, but by Ixr1 binding to the $R N R 1$ promoter upon genotoxic stress. This Ixr1-dependent regulation of $R N R 1$ is independent of Dun1 but requires Rad53 (Tsaponina et al., 2011). Another Rad53-dependent, Dun1-independent regulation of $R N R 1$ involves phosphorylation-dependent dissociation of Nrm1 from MBF (Travesa et 68 al., 2012).

In addition to upregulating the dNTP pools, defects in cells lacking Rad53 can be suppressed by manipulating factors functioning in transcription regulation, cell wall maintenance, proteolysis and cell cycle control (Desany et al., 1998; Manfrini et al., 2012). Moreover, Rad53 kinase targets and interaction partners found in biochemical and proteomic studies suggests that the kinase is pleiotropic 
In this study, while investigating of the response of yeast cells to replication stresses caused by depletion of dNTPs, we found that Rad53 not only binds to sites of DNA synthesis, but it localized to more than $20 \%$ of gene promoters in the S. cerevisiae genome, suggesting a global role in coordinating stress responses. Furthermore, we provide evidence that untimely activation of replication from late origins can negatively affect transcription activity of nearby genes.

\section{Results}

\section{Initiation, elongation and recovery of DNA replication in checkpoint mutants}

DNA replication in the presence of low dNTP levels was examined by releasing G1-phase cells for either 45 (HU45) or 90 (HU90) minutes into media containing hydroxyurea (HU), coupled with labeling DNA synthesis with 5-ethynyl-2'deoxyuridine (EdU) (Sheu et al., 2016, 2014). The purified EdU-DNA was subjected to high-throughput DNA sequencing and the reads were mapped to the genome, yielding replication profiles for wild-type (WT) and three DNA damage checkpoint mutants, rad53 ${ }^{K 227 A}$ (a kinase-deficient version of Rad53), $m r c 1 \Delta$ (null for Mrcl mediator of the DRC branch) and $\operatorname{rad} 9 \Delta$ (null for Rad9 mediator of DDC branch).

In WT cells, DNA synthesis occurred only from early origins because of the activated DRC checkpoint, which inhibits late origin firing (Figure 1a, [HU90] and Figure 1-figure supplement 1a [HU45 and HU90] $)^{5,6}$. As expected DNA synthesis was readily detected from late origins (red arrows) in the kinase-deficient $r a d 53^{K 227 A}$ and $m r c l \Delta$ mutants. In contrast, the rad9 $\Delta$ mutant profile appeared identical to that of WT (Figure 1a). Thus, the DRC branch (Mrc1), but not the DDC branch $(\operatorname{Rad} 9)$, represses late origin firing in response to this replication stress.

Among the 829 active or potential origins of DNA replication (Siow et al., 2012), 256 origins are active in WT cells and 521 origins are active in the $\mathrm{rad} 53^{K 227 A}$ and $m r c 1 \Delta$ mutants, specifying "early" (E) and "late" (L) firing origins, respectively. The remaining 308 were therefore inactive (I) under these conditions. The EdU peak signals in each mutant for these origin categories shows that the rad53 ${ }^{K 227 A}$ mutant favored late origins over early origins (Figure $1 \mathrm{~b}$ ), which was particularly prominent in heterochromatic regions on chromosome III, such as $H M R, H M L$ and telomere-proximal regions harboring very late firing origins (Figure 1-figure supplement $1 \mathrm{~b}$ ). This pattern is not seen in the $m r c 14$, suggesting that it is due to loss of Rad53 kinase activity but not DRC signaling.

Rad53 is required for stability of DNA stalled replication forks (Bacal et al., 2018; Kumar and Huberman, 2008; Lopes et al., 2001; Seiler et al., 2007; Tercero et al., 2003), which was confirmed by labelling DNA synthesis during recovery from HU-induced replication stress. Cells that progressed from G1 - into S-phase in HU for 45 min. were released from the HU block and DNA synthesis labeled with EdU during an additional $25 \mathrm{~min}(\mathrm{HU} \rightarrow \mathrm{S} 25)$ or were continued in $\mathrm{HU}$ for another $45 \mathrm{~min}$. and labeled with EdU (HU $\rightarrow \mathrm{HU} 45)$ (Figure 1c). In WT cells, DNA synthesis during recovery from $\mathrm{HU}$ continued from the stalled replication forks (Figure 1d). For very efficient early origins, such as ARS305, ARS306 and ARS307 (Figure 1d, black arrows), little new synthesis occurred at origins during the recovery, suggesting efficient initiation at these origins. In contrast, for moderately early origins, such as ARS309 and ARS315 (Figure 1d, brown arrows), DNA synthesis occurs at both the origin and recovered forks in the cell population. DNA synthesis from late origins 
is not detectable (Figure 1d, red arrows). Thus, in WT, DNA synthesis during recovery from replication stress continued mainly from already activated replisomes that had progressed away from origins. If the replication stress persisted, DNA synthesis continued slowly only from existing replisomes (Figure 1d).

In the recovering $m r c 1 \Delta$ mutant, DNA synthesis continued from stalled replisomes, albeit slowly, but unlike WT, new initiation at efficient early origins, such as ARS305, ARS306 and ARS307 was also detected (Figure 1d), suggesting that Mrc1 is important for efficient initiation at early origins in addition to its established role in stimulating fork progression (Osborn and Elledge, 2003; Tourrière et al., 2005; Yeeles et al., 2016). During recovery from stress, the rad53 $3^{K 227 A}$ mutant failed to restart DNA synthesis at most stalled forks, except for the replicons in the heterochromatic regions, where new initiation was also detected (Figure 1d). Thus, the replication fork collapse was more severe in the absence of Rad53 kinase compared to the absence of checkpoint signaling in the mrcl $\Delta$ mutant.

\section{Rad53 is recruited to sites of DNA synthesis independent of checkpoint signaling}

To investigate the status of replisomes, chromatin immunoprecipitation and deep sequencing (ChIPseq) was employed to follow localization of Cdc45, which is associated with activated helicases at the replisomes. G1 arrested cells and cells released for 45 and 90 min. in HU were processed for ChIPseq analysis (Behrouzi et al., 2016).

Using either normalized read counts or a heatmap analysis around active origins that are ranked in order of DNA replication timing ${ }^{28}, \mathrm{Cdc} 45$ in WT cells was found moving only from early origins, (Figure 2a and 2b; early origins in top panel and late origins in bottom panel). In contrast, Cdc45 is present at both early and late origins in both $\mathrm{rad} 53^{K 227 A}$ and $\mathrm{mrcl} \Delta$ mutants, with slower progression in the mrc1 $\Delta$ mutant (Figure 2b; Figure 2- figure supplement 1a), consistent with its role in progression at replication forks (Tourrière et al., 2005; Yeeles et al., 2016). Cdc45 in the rad53 ${ }^{\text {K227A }}$ mutant emanating from late origins continued to move from HU45 to HU90, whereas at the early origins Cdc45 signal did not move further away for origins (Figure 2b). Since Cdc45 can recruit Rad53 to restrict CMG helicase activity (Can et al., 2018; Devbhandari and Remus, 2020), the limited Cdc45 signal at early origins here suggests that, in the absence of active Rad53 kinase, replisomes departed from origins but disintegrated. The persistent signal at origins in HU90 is consistent with firing at early origins in those cells that had not initiated DNA replication during the HU block.

Phosphorylation of histone $\mathrm{H} 2 \mathrm{~A}$ at serine 129 (S129; $\gamma$-H2A) by the sensor kinase Mec1 is an indication of checkpoint activation. $\gamma$-H2A ChIP-seq monitors the genome distribution of checkpoint activation under HU stress (Figure $2 \mathrm{c}$ and d; Figure 2 - figure supplement $1 \mathrm{~b}$ ). In WT cells, $\gamma-\mathrm{H} 2 \mathrm{~A}$ signals are particularly high around the earliest firing origins in HU45 and HU90, suggesting that stress signals emit mostly from early origins. In the rad53 $3^{K 227 A}$ and $m r c 1 \Delta$ mutants, $\gamma-\mathrm{H} 2 \mathrm{~A}$ is found at both early and late origins, however, in the $\operatorname{rad} 53^{K 227 A}$ mutant, the signal at early firing origins reduces with time, suggesting that Rad53 kinase activity is needed to maintain stress signaling by $\mathrm{Mec} 1$ at early origins. In contrast, the Mrc1 is not strictly required to induce or maintain $\gamma-\mathrm{H} 2 \mathrm{~A}$. 
Interestingly, $\gamma-\mathrm{H} 2 \mathrm{~A}$ is observed at genomic regions surrounding the very late origins in G1-phase in both WT and mutants (Figure $2 \mathrm{~d}$ and Figure 2 - figure supplement 1b). It is possible that these $\gamma$ $\mathrm{H} 2 \mathrm{~A}$ signals reflect a low level of ssDNA gaps at these late-replicating regions that was tolerated and carried over from the previous cell cycle, similar to unrepaired post-replication gaps resulting from low level of UV irradiation in S. pombe G2-phase (Callegari and Kelly, 2006).

Rad53 kinase detected by ChIP-seq at genome sites in WT cells largely follows the progression of replication forks (Figure 2e and f; Figure 2 - figure supplement 1c). Rad53 is also detected at late origins in both checkpoint mutants, but dispersed at late times in the rad53 ${ }^{K 227 A}$ mutant. The spreading of Rad53 signal in the mrcl $\Delta$ mutant is more restricted, consistent with slower replication fork progression. Surprisingly, Rad53 binding to replication forks does not require the Mrc1, suggesting checkpoint-independent recruitment of Rad53 to sites of DNA synthesis.

\section{Rad53 binds to promoters of genes involved in multiple cellular processes}

Unexpectedly, we noticed many Rad53 peaks even in G1 arrested cells (Figure 2e) and many of these peaks localized upstream of transcription start sites (TSS) or promoters (Figure 3). In WT, some peak signals change as cells progress from G1-phase into HU arrested S-phase. For example, Rad53 at the RNR1 promoter increases from G1 to HU45 and HU90 (Figure 3a and b). A similar pattern occurs at the RNR3 promoter. The Rad53 signal at promoters are present in both $\mathrm{rad} 53^{K 227 A}$ and $m r c 1 \Delta$ mutants (Figure 3a). Rad53 binding to promoters also occurs in the smll null mutant (smll $\Delta$ ) and the mecl null mutant (mecl $\Delta$ sml1 $\Delta$ ), but is absent in rad53 null (rad53 $\Delta$ smll $\Delta$ ), demonstrating antibody specificity (Figure 3b and Figure 3 - figure supplement 1a). Thus, both the sensor kinase Mec 1 and Mrc1 are not required for the recruitment of Rad53 to these sites.

Whole genome analysis shows that $\sim 90 \%$ of the Rad53 peaks are either upstream of or overlap the TSS (Figure 3 - figure supplement 1b). Rad53 promoter binding is temporally dynamic in a subset of genes, suggesting regulation by cell cycle progression or DNA replication stress. Heatmaps of the Rad53 signals at $2 \mathrm{~kb}$ intervals centered on all transcription start sites (TSS) show a global trend of increasing Rad53 binding as cells progress from G1-phase into HU45 or HU90 (Figure 3c), concomitant with increased levels of Rad53 protein in cells treated with HU (Figure 3 - figure supplement 2a). The increase parallels entry into S-phase, as measured by Orc6 phosphorylation, destruction of Sml1 and histone H2A phosphorylation (Figure 3 - figure supplement 2a-c). Additional genes show increased Rad53 binding as cells progress from G1- into S-phase (Figure 4a, upper panels), but at other promoters Rad53 binding decreases during the same time course (Figure 4a, lower panels). However, at most genes Rad53 remains constant. and mrcl $\Delta$ mutants (CP set), and based on the type of genes that bind Rad53, in transcription factor mutants ixrl $\Delta$, swi4 $\Delta$, swi6 $\Delta$ and WT (TP set). Residual analysis in WT identified the top differentially binding (DB) genes (Figure 4b, Figure 4c for CP and TP sets; orange dots). Among the top $1000 \mathrm{DB}$ from each set, 435 genes were identified in both (Figure 4b, 435 Top DB overlap). Overall, during the G1- to S-phase transition (HU45), there are more genes with increased Rad53 promoter binding than those with decreased binding. Many of these genes encode proteins involved in cell cycle progression (e.g., cyclins and regulators of DNA replication) and cell growth (e. g., cell 210 wall maintenance and mating response). 
In the $\operatorname{rad} 53^{K 227 A}$ mutant, the increase in Rad53 promoter binding is transient and generally weaker, consistent with lower protein levels (Figure 3c). In the mrcl $\Delta$ mutant, the binding at the RNRl promoter is reduced compared to WT, despite an increase Rad53 protein (Figure 3a, Figure 3 - figure supplement 2a). In contrast, the increase in Rad53 binding at the PCL1 promoter appears to be less affected by the checkpoint mutations (Figure 3a). Thus, the DRC checkpoint only affects differential binding of Rad53 to a subset of promoters. At other promoters, cell cycle progression or response to mating pheromone due to treatment and removal of $\alpha$-factor may contribute to differential $\operatorname{Rad} 53$ promoter binding.

Visual inspection of the ChIP-Seq peaks suggested that Rad53 bound to numerous gene promoters and TSSs throughout the genome. Rad53 ChIP-Seq was compared to a previous ChIP-Seq data set of the sequence-specific transcription factor Swi6, part of SBF and MBF that control cell-cycle regulated genes (Breeden, 2003). The Gini indices computed for Swi6 and two of our Rad53 replicates are 0.763, 0.2918, and 0.2982, respectively, calculated from Lorenz curves (Figure 3d). Rad53 has a higher coverage for many promoters while Swi6, as expected, shows substantially high coverage only for a limited number of promoters.

Previous studies have found that under certain conditions, regions of the genome are promiscuously present in ChIP-Seq studies independent of the antibody used, enriching for sequences in and around gene bodies of highly expressed genes (Park et al., 2013; Teytelman et al., 2013). We therefore examined whether these gene regions were promiscuously present under our conditions. The ChIPSeq data using anti- $\gamma \mathrm{H} 2 \mathrm{~A}$ antibodies did not enrich for sequences at the TSS, whether or not the highly enriched sequences observed by Teytelman et al. and Park et al. were included in the analysis (Figure 3 - figure supplement 3). In addition, analysis of the Rad53 antibody ChIP with or without the highly enriched sequences observed by Teytelman et al. and Park et al. did not alter the pattern or frequency of Rad53 binding to TSSs (Figure 3 - figure supplement 4a and 4b). When we specifically examined at the pattern of Rad53 antibody enrichment at these 296 highly enriched genes we did not observe localization to TSSs, but enrichment to gene bodies as previously reported (Park et al., 2013; Teytelman et al., 2013) (Figure 3 figure supplement 4c). KEGG analysis of the genes enriched in the studies by Teytelman et al. and Park et al. show predominantly genes encoding snoRNAs and tRNAs, genes we did not find in the promoter binding for Rad53 (Figure 3 - figure supplement 5; see below). Finally, we did not see any gene enrichment when RAD53 was deleted from the strain (Figure 3 figure supplement 1a). Thus, we suggest that the Rad53 binding observed here is not the same as the promiscuous, non-specific enrichment of genome regions reported by Teytelman et al. and Park et al. Moreover, Rad53 binding to promoters is transcription factor dependent (see below).

\section{The relationship between Rad53 promoter binding and gene expression}

The relationship between Rad53 promoter recruitment was compared to gene expression from RNAseq analysis using the same conditions. RNA-seq replicates from 4 strains ( WT, $\operatorname{rad} 9 \Delta, \operatorname{rad} 53^{K 227 A}$ and $m r c 1 \Delta$ ), each with 3 stages (G1, HU45 and HU90) were analyzed using rank data analysis (Figure 5a). The expression profiles in G1 are very similar among all strains. In HU, however, two groups are evident; rad9 $\Delta$ is like WT since Rad9 has no role in the DRC checkpoint branch. In contrast, rad53 ${ }^{K 227 A}$ and $m r c 1 \Delta$ cluster together in both HU45 and HU90, consistent with Rad53 and Mrc1 functioning together in the response to HU stress. 
In the hierarchical clustering, cell cycle stage contributes more to similarities than the genotype (Figure 5a). Pair-wise comparison of G1 to HU45 in WT and rad9 4 cells shows that 2300 genes exhibited significant expression changes (differentially expressed genes; DEGs; Figure 5b). The number of DEGs increases further to $~ 3000$ when comparing G1 to HU90. In both rad53 ${ }^{K 227 A}$ and mrcl $\Delta$ mutants, 2500 DEGs are detected from G1 to HU45, which increases to >3400 in G1 to HU90. The response to cell cycle stage is largely equally distributed between up and down regulation. A WT and rad9 $\Delta$ comparison shows only 5 DEGs, demonstrating that Rad9 does not contribute to gene expression changes under HU stress.

The overall heatmap signal of Rad53 upstream of TSSs is higher in the significant DEGs than in the insignificant DEGs, suggesting that Rad53 may play a role in control of gene expression (Figure 5c and Figure 5 - figure supplement 1). Gene co-expression analysis of the RNA-seq data yields ten coexpression clusters of DEGs in WT (G1 $\rightarrow$ HU45) (Figure 6a and Figure 6 - figure supplement 1). Specific, dynamic Rad53 binding at promoter regions occurs in most clusters (Figure 6b), with GO functions including cell cycle regulation, mating response, proteolysis, transport, oxidation-reduction process and organic acid metabolism (Figure 6a).

Within the 435 Top DB overlapping genes (Figure 4b), 236 show significant expression changes. Plots of Rad53 binding changes against gene expression changes of these 236 genes show a positive correlation between Rad53 binding change and gene expression change (Figure 6c, left panel). Among this group, 51 out of 54 genes with decreased Rad53 signal are down-regulated in mRNA levels. Genes with increased Rad53 signals are partitioned between up-regulation and downregulation (108 and 74, respectively). Further break down of the 236 gene group into co-expression clusters of the DEGs in WT (G1 $\rightarrow$ HU45) revealed that genes in clusters 1 and 7 exhibit the strongest correlation between Rad53 binding and gene expression changes (Figure 6c). Thus, specific subsets of DEGs in the shift from G1 $\rightarrow$ HU exhibit correlations between a change in gene expression and Rad53 promoter binding.

\section{Checkpoint mutants cause down-regulation of gene expression near promiscuously active late origins}

Upon inspection of Rad53 heatmaps around TSSs, we noticed that in several co-expression clusters from the DEGs in the HU45 ( $m r c 1 \Delta$ vs WT) comparison (Figure 7a), down-regulated genes tend to have a strong Rad53 signal not only upstream of the TSS, but a broad signal within gene bodies (Figure 7b). This pattern is prominent in the mrcl $\Delta$ mutant at HU45 and further intensifies in HU90. The gene body localization is also found transiently in $\mathrm{rad} 53^{K 227 A}$ cells (Figure 7 - figure supplement 1). Such a gene body signal is not as prevalent in the WT HU45 and HU90 samples. Since Rad53 is also recruited to active origins and moves with the replication fork, we suspected these gene body signals in the checkpoint mutants may be caused by the promiscuous activation of near-by origins that are normally inactive in WT, creating conflicts between DNA replication and gene transcription. The transient nature of the Rad53 localization at gene body in this group of genes in the $\operatorname{rad53} 3^{K 227 A}$ mutant is also consistent with the transient signal pattern at these late origins (Figure $2 \mathrm{f}$, bottom panel). Thus, we investigated the relationship between these genes and their closest replication 
The distance of replication origins to the nearest TSS, the relative orientation of the gene to the origin (head-on or co-directional) and the origin type (early, late or inactive; Figure 1b) was determined and correlated with the DEG clusters (Figure 7c). Overall, most of the down regulated genes in cluster 1 of this group are situated very close to active origins $(<2 \mathrm{~kb}$ between origin center and TSS, light purple marks and $<1 \mathrm{~kb}$, dark purple marks). Interestingly, the pattern of origin to promoter distance marks largely mirrored the patten of the Rad53 ChIP signal within the gene bodies (Figure 7b and 7c). This correlation pattern is not found in the WT ChIP heatmap. Within the DEG group, genes situated $5 \mathrm{~kb}$ or more away from closest active origins are similarly distributed between up regulation and down regulation of gene expression (Figure 7d, left panels). However, for those genes that are closer to an active origin, the bias to be down regulated gene increases. For those that gene situated less than $1 \mathrm{~kb}$ away from active origins, more than $80 \%$ are down-regulated genes.

The DEGs in HU45 ( $m r c 1 \Delta$ vs $W T$ ) that are more than $5 \mathrm{~kb}$ away from active origins are also similarly distributed between up and down regulation (Figure 7d, middle panels). More down regulated genes are found when the nearby origins are active. The bias is even stronger for genes that are close to late origins, which become active in $\mathrm{HU}$ when Mrc1 is absent. Because late origins and intermediate early origins are more active in the $\operatorname{mrcl} \Delta$ mutant, it is possible that nearby gene expression is negatively affected by active DNA synthesis. Furthermore, the bias toward the down regulation is even stronger ( $>80 \%)$ when the nearby origin is in a head-on orientation towards the gene (Figure 7d, right panels). Similarly, a bias exists toward down regulation of DEGs from HU45 ( $\operatorname{rad} 53^{K 227 A}$ vs WT) that are close to active origins (Figure 7 - figure supplement 1 ). The tendency to find a high Rad53 signal at gene bodies in the $m r c 1 \Delta$ and $r a d 53^{K 227 A}$ mutants also occurred in the down-regulated DEGs in $m r c 1 \Delta(\mathrm{G} 1 \rightarrow$ HU45) (Figurer 5 - figure supplement $1 \mathrm{c}$ ), likely caused by the same proximal origins. Thus, the untimely activation of replication origins in the checkpoint mutants affects gene expression and Rad53 binding to gene bodies.

\section{Rad53 binding changes coincide with the changes in gene expression for targets of cell cycle regulators $\mathrm{SBF}, \mathrm{MBF}$ and mating response regulator Ste12}

The DEGs in WT (G1 $\rightarrow$ HU45) were associated with co-expression clusters that showed a strong correlation between Rad53 binding and gene expression (Figure 6c, clusters 1 and 7). They contain genes that encode targets of SBF and MBF, key transcription factor complexes comprised of a shared regulatory subunit, Swi6 and the DNA-binding subunits Swi4 and Mbp1, respectively (Breeden, 2003). Their target genes include multiple G1- and S-phase cyclin genes, such as PCL1, CLN1, CLN2, CLB5, CLB6. Evidence suggests that SBF and MBF are directly regulated by Rad53 kinase (Oliveira et al., 2012; Sidorova and Breeden, 2003; Travesa et al., 2012) and Rad53 may regulate expression of targets of Msn4, Swi6, Swi4, and Mbp1 through Dun1-independent mechanisms (Jaehnig et al., 2013). Thus, we analyzed the annotated targets of these transcription factors compiled in the Saccharomyces Genome Database (SGD; https://www.yeastgenome.org). Among the 81 genes that are candidate targets for both Swi4 and Swi6, 36 genes were found in the 236 significant DEGs in the Top DB overlap (Figures $4 b$ and $6 c$ ) with an enrichment of 12.91. Scatter plot comparisons of Rad53 binding and gene expression changes of these 36 genes show a clear positive correlation (Figure $8 \mathrm{a}$, SBF top panel). Combining the data from the checkpoint mutants (Figure 8a, SBF bottom panel and Figure 8 - figure supplement 1a) show that most of these genes have similar levels of differential expression in the rad9 $\Delta$ mutant compared with WT from G1 to HU45, whereas in the $m r c 1 \Delta$ and rad53 $3^{K 227 A}$ mutants exhibit different level of changes. Similar plot patterns were found with 26 out of 
65 MBP targets with an enrichment of 11.62 (Figure 8a and Figure 8 - figure supplement $1 \mathrm{~b}$ ), including overlap between the targets of SBF and MBF (19 genes). We also found enrichment for targets of transcription factor Msn4 and patterns of correlation (Figure 8a and Figure 8 - figure supplement 1d, Msn4 panels), including 12 out of 22 Msn4 targets that are also SBF targets.

Many of the genes with decreased Rad53 binding at the promoters are mating response genes (Figures $4 \mathrm{~b}$ and $\mathrm{c}$ ). Therefore, the targets of Ste12, a key transcription factor activated by MAPK signaling to activate genes involved in mating or pseudohyphal/invasive growth pathways were investigated. Of 183 potential targets of Ste 12 annotated in SGD, 34 are in the 236 significant DEGs in the Top DB overlap (Figures 6c and 8a). All the Ste12 targets that have decreased Rad53 binding are down regulated as cells entered S-phase. Moreover, 20 out of the 34 Ste12 targets in the Top DB group show increased Rad53 binding in HU and 11 of these 20 genes are also targets of SBF. Thus, regulation by SBF appears to be responsible for the correlation between increased Rad53 binding at the promoter and up-regulation of these target genes.

\section{SBF plays a major role in the localization of Rad53 to the promoters of its target genes under replication stress}

To determine the contribution of various transcription regulators in recruitment of Rad53 to gene promoters, Rad53 ChIP-seq analysis in WT, ixrl $\Delta$, swi4 $\Delta$ and swi6 $\Delta$ mutants was performed. In the scatter plot of the Rad53 signal upstream of TSSs in G1 versus HU45 from the WT sample, SBF targets in the Top DB (Figure 8b, orange/red diamonds) showed significant deviation from the global trend (blue dots). In swi4 $\Delta$ and swi6 $\Delta$ mutants, the signal for all of these SBF targets collapses towards the global trend (purple and light olive dots, swi6 $\Delta$ and swi4 $\Delta$, respectively), suggesting that Rad53 signal changes at these genes depends on SBF. In the ixr1 $\Delta$ mutant (green dots), the majority of these SBF targets remain deviated from the global trend in the scatter plot, except for the RNR1 gene, indicated in the close-up plots (Figure $8 \mathrm{~b}$, lower panels), whose position collapsed in all three mutants. Rad53 binding to the RNR1 promoter is reduced in both SBF mutants, consistent with $R N R 1$ being a target of SBF and MBF ((Bruin et al., 2006)). Rad53 binding is completely eliminated from the TOS6 (target of $\underline{\mathrm{SBF}} 6$ ) promoter while for PCL1 and YOX1, both targets of SBF, Rad53 binding does not increase in HU. Interestingly, at the promoter of $R N R 3$, the paralog of $R N R 1$, Rad53 binding in the SBF mutants is low, even though $R N R 3$ may not be a SBF or MBF target. On the other hand, ixrl $\Delta$ reduces Rad53 binding to RNR1 in HU but has no effect on Rad53 recruitment at the RNR3 promoter (Figure 8c).

\section{Discussion}

Following hydroxyurea induced replication stress, Rad53 was recruited to active origins of DNA replication and to DNA replication forks in a checkpoint independent manner since mrcl $\Delta$ and rad53 ${ }^{K 227 A}$ mutants had little effect on binding. Rad53 is targeted to replisomes by the helicase subunits Cdc45 and Mcm2 where it is activated by Mec1 kinase dependent on Mrc1 at the fork, and stabilizes the replisome (Can et al., 2018; Cobb et al., 2005; Lou et al., 2008; McClure and Diffley, 2021; Szyjka et al., 2008). Maintenance of Rad53 at the replication forks requires Rad53 kinase activity but not DRC checkpoint signaling. Since Rad53 kinase can auto-activate itself (Gilbert et al., 2001; Lanz et al., 2019; Pardo et al., 2017; Saldivar et al., 2017), we suggest that either auto- 
activation or binding to a phosphorylated replisome protein is required for the continued presence of Rad53 at replication forks.

Checkpoint signaling also prevents replication initiation in late replicating regions of the genome (Hamperl and Cimprich, 2016). However, in the checkpoint mutants, these late origins become active and Rad53 was recruited to the body of origin proximal genes. Concomitantly, gene expression of these genes was reduced, perhaps mediated by recruitment of Rad53. We suggest that the normal temporal order of replication of the genome throughout S-phase has evolved to prevent conflicts between replication and transcription, which is particularly important in a gene dense genome such as S. cerevisiae. It is known that late replicating genes are tethered to the nuclear pore complexes in the nuclear periphery and checkpoint signaling, including Rad53 kinase, is required for preventing topological impediments for replication fork progression (Bermejo et al., 2011; Hamperl and Cimprich, 2016). Moreover, during normal replication, Mec1 may locally activate Rad53 to deal with difficult to replicate regions or regions of replication-transcription conflict without triggering full blown checkpoint activation (Bastos de Oliveira et al., 2015). Rad53 kinase inhibits Mrc1 stimulation of the CMG helicase (McClure and Diffley, 2021), consistent with our observation that replication fork progression is limited in the absence of Mrc1 and that replication forks cannot be rescued after DNA damage in rad53 ${ }^{K 227 A}$ cells (Forey et al., 2020).

Unexpectedly we also found Rad53 constitutively bound to $>20 \%$ of the gene promoters in the yeast genome, independent of Mrc1 and Rad53 kinase activities. The genes encode proteins with diverse activities, including various aspects of cell cycle, metabolism, protein modification, ion transport, cell wall organization and cell growth. The levels of Rad53 binding to most of these genes did not change during the time course in HU, whereas Rad53 binding increased at promoters for genes such as $R N R 1$, $R N R 3$ and TOS6. In contrast, Rad53 levels decreased on the promoters of genes involved in response to mating pheromone as cells exited from $\alpha$-factor induced G1 arrest into the cell division cycle. The prevalent and dynamic changes in Rad53 promoter-bound levels did not necessarily depend on checkpoint signaling at genes like PCL1, but in some cases such as $R N R 1$, the increase in $\operatorname{Rad} 53$ levels was reduced in checkpoint mutants.

The conditions employed in this study, cell cycle entry in the presence of hydroxyurea, may determine the nature of the genes that display dynamic binding of Rad53 to gene promoters. It is known that Rad53 phosphorylates transcription factors such as the SBF and MBF subunit Swi6 and the MBF co-repressor Nrm1 (Sidorova and Breeden, 2003; Travesa et al., 2012) and that Irx1controls transcription of RNR1 (Tsaponina et al., 2011). Removal of Swi4, Swi6 or Ixr1 reduced, and in some cases eliminated Rad53 binding to promoters of genes controlled by these transcription factors. Rad53 bound to the Nrm1 promoter, suggesting an additional regulation of cell cycle-dependent transcription control by Rad53. Rad53 also bound to promoters of genes encoding histones H3 and $\mathrm{H} 4$, suggesting that in addition to its known role in histone degradation (Gunjan and Verreault, 2003) Rad53 controls histone gene expression. This is consistent with previous findings that Rad53 targets Yta7 (Smolka et al., 2006), which interacts with FACT to regulate histone gene expression and inhibits Spt $21^{\text {NPAT }}$-regulated histone genes expression (Bruhn et al., 2020; Gradolatto et al., 2008). In the absence of Rad53 protein, histone levels become elevated, causing global effects on gene expression (Bruhn et al., 2020; Tsaponina et al., 2011). 
Our data is consistent with the possibility that the Rad53 kinase contributes to the transcriptional regulation as a structural component, as previously suggested for several MAP kinases (Alepuz et al., 2001; Kim et al., 2008; Sanz et al., 2018). Like the stress induced kinase Hog1, Rad53 binding to promoters may be dynamic in other stress conditions, which is under investigation. A major unanswered question is how does Rad53 bind to so many diverse promoter sites.

\section{Acknowledgements}

This research was supported by NIH grants R01GM45436 and R01LM012736 and a gift from the Goldring Family Foundation. The Cold Spring Harbor Laboratory Cancer Center supported core research resources (P30-CA045508). RKK was supported by Uehara Memorial Foundation Postdoctoral Fellowship.

\section{Materials and methods}

\section{Yeast strains and methods}

Yeast strains generated in this study were derived from W303-1a (MATa ade2-1 can1-100 his3-11,15 leu2-3,112 trp1-1 ura3-1) and are described in Supplemental Table 1. All the yeast strains used for the whole-genome DNA replication profile analyses have a copy of the BrdU-Inc cassette inserted into the URA3 locus ((Viggiani and Aparicio, 2006)). For G1 arrest of bar1 $\Delta$ strains, exponentially growing yeast cells $\left(\sim 10^{7}\right.$ cell/mL) in YPD were synchronized in G1 with $25 \mathrm{ng} / \mathrm{mL}$ of $\alpha$-factor for $150 \mathrm{~min}$ at $30^{\circ} \mathrm{C}$. For G1 arrest of $B A R 1$ strains, exponentially growing cells were grown in normal YPD, then transferred into YPD (pH3.9), grown to $\sim 10^{7}$ cell $/ \mathrm{mL}$, and then synchronized in G1 with three doses of $\alpha$-factor at $2 \mu \mathrm{g} / \mathrm{mL}$ at $0-, 50$-, and 100 -min time point at $30^{\circ} \mathrm{C}$. Cells were collected at $150 \mathrm{~min}$ for release. To release from G1 arrest, cells were collected by filtration and promptly washed twice on the filter using one culture volume of $\mathrm{H} 2 \mathrm{O}$ and then resuspended into YPD medium containing $0.2 \mathrm{mg} / \mathrm{mL}$ pronase E (Sigma).

\section{Protein sample preparation and immunoblot analysis}

TCA extraction of yeast proteins was as described previously ((Sheu et al., 2014)). For immunoblot analysis, protein samples were fractionated by SDS-PAGE and transferred to a nitrocellulose membrane. Immunoblot analyses for Orc6 (SB49), Rad53 (ab104232, Abcam), $\gamma$-H2A (ab15083, Abcam) and Sml1 (AS10 847, Agrisera) were performed as described ((Sheu et al., 2016, 2014)).

\section{Isolation and preparation of DNA for whole-genome replication profile analysis}

Modified protocol based on previously described ((Sheu et al., 2016, 2014)). Briefly, yeast cells were synchronized in G1 with $\alpha$-factor and released into medium containing $0.2 \mathrm{mg} / \mathrm{mL}$ pronase $\mathrm{E}$, $0.5 \mathrm{mM}$ 5-ethynyl-2' -deoxyuridine (EdU) with or without addition of $200 \mathrm{mM}$ HU as indicated in the main text. At the indicated time point, cells were collected for preparation of genomic DNA. The genomic DNA were fragmented, biotinylated, and then purified. Libraries for Illumina sequencing were constructed using TruSeq ChIP Library Preparation Kit (Illumina). Libraries were pooled and submitted for $50 \mathrm{bp}$ paired-end sequencing. modification. About $10^{9}$ synchronized yeast cells were fixed with $1 \%$ formaldehyde for 15 min at room temperature (RT), then quenched with $130 \mathrm{mM}$ glycine for $5 \mathrm{~min}$ at RT, harvested by 
centrifugation, washed twice with TBS (50 mM Tris. $\mathrm{HCl}$ pH 7.6, $150 \mathrm{mM} \mathrm{NaCl}$ ), and flash frozen. Cell pellets were resuspended in $600 \mu \mathrm{l}$ lysis buffer $(50 \mathrm{mM}$ HEPES-KOH pH 7.5, $150 \mathrm{mM} \mathrm{NaCl,} 1$ mM EDTA, $1 \%$ Triton X-100, 0.1\% Na-Deoxycholate, 0.1\% SDS, 1 mM PMSF, protease inhibitor tablet (Roche)), and disrupted by bead beating using multi-tube vortex (Multi-Tube Vortexer, Baxter Scientific Products) for 12-15 cycles of 30 seconds vortex at maximum intensity. Cell extracts were collected and sonicated using Bioruptor (UCD-200, Diagenode) for 38 cycles of pulse for 30 seconds "ON", 30 seconds "OFF" at amplitude setting High (H). The extract was centrifuged for 5 min at 14,000 rpm. The soluble chromatin was used for IP.

Antibodies against Cdc45 (CS1485, this lab (Sheu and Stillman, 2006)), Rad53 (ab104232, Abcam), $\gamma$-H2A (ab15083, Abcam) was preincubated with washed Dynabeads Protein A/G (Invitrogen, 1002D and 1004D). For each immunoprecipitation, $80 \mu \mathrm{l}$ antibody-coupled beads was added to soluble chromatin. Samples were incubated overnight at $4^{\circ} \mathrm{C}$ with rotation, after which the beads were collected on magnetic stands, and washed 3 times with $1 \mathrm{ml}$ lysis buffer and once with $1 \mathrm{ml} \mathrm{TE}$, and eluted with $250 \mu \mathrm{l}$ preheated buffer $(50 \mathrm{mM}$ Tris. $\mathrm{HCl} \mathrm{pH} 8.0,10 \mathrm{mM}$ EDTA, $1 \%$ SDS $)$ at $65^{\circ} \mathrm{C}$ for $15 \mathrm{~min}$. Immunoprecipitated samples were incubated overnight at $65^{\circ} \mathrm{C}$ to reverse crosslink, and treated with $50 \mu \mathrm{g}$ RNase A at $37^{\circ} \mathrm{C}$ for $1 \mathrm{hr} .5 \mu \mathrm{l}$ proteinase $\mathrm{K}$ (Roche) was added and incubation was continued at $55^{\circ} \mathrm{C}$ for $1 \mathrm{hr}$. Samples were purified using MinElute PCR purification kit (Qiagen). Libraries for Illumina sequencing were constructed using TruSeq ChIP Library Preparation Kit (Illumina, IP-202-1012 and IP-202-1024).

The duplicate Rad53 ChIP-Seq data was compared to published ChIP-Seq data for Swi6 (Park et al., 2013) (SRX360900: GSM1241092: swi6_DMSO_illumina; Saccharomyces cerevisiae; ChIP-Seq), creating Gini indexes from calculated Lorenz curves (Andri et mult. al. S (2021). DescTools: Tools for Descriptive Statistics. R package version 0.99.41, https://cran.r-project.org/package=DescTools).

\section{Sample preparation for RNA seq}

About $2-3 \times 10^{8}$ flash-frozen yeast cells were resuspended in Trizol (cell pellet: Trizol = 1:10) and vortex for $15 \mathrm{sec}$ and incubate $25^{\circ} \mathrm{C}$ for $5 \mathrm{~min}$. Add $200 \mu \mathrm{l}$ chloroform per $1 \mathrm{ml}$ of Trizol-cell suspension, vortex $15 \mathrm{sec}$, then incubate at room temp for $5 \mathrm{~min}$ and centrifuge to recover the aqueous layer. The RNA in the aqueous layer were further purified and concentrated using PureLink Column (Invitrogen, 12183018A). The RNA was eluted in $50 \mu 1$ and store at $20^{\circ} \mathrm{C}$ if not used immediately. Store at $-80^{\circ} \mathrm{C}$ for long term. Paired-end RNA-seq libraries were prepared using TruSeq stranded mRNA library preparation kit (Illumina, 20020594).

\section{Generation of coverage tracks using the Galaxy platform}

For visualization of read coverage in the Integrated Genome Browser ((Freese et al., 2016)), the coverage tracks were generated using the Galaxy platform maintained by the Bioinformatics Shared Resource (BSR) of Cold Spring Harbor Lab. The paired-end reads from each library were trimmed to 31 bases and mapped to sacCer3 genome using Bowtie ((Langmead, 2010)). The coverage track of mapped reads was then generated using bamCoverage ((Ramírez et al., 2014)) with normalization to $1 \mathrm{x}$ genome.

\section{Definition of the origin-types}


530 Based on the BamCoverage output for EdU signal in $W T$, rad53 ${ }^{K 227 A}$ and $m r c 1 \Delta$, we categorized 829 531 origins listed in the oriDB database ((Siow et al., 2012)). We define the early origins as the one whose 532 signal at the first time point is larger than 2. The late origins are extracted from the rest of the origins if the average signal value at the later time point is larger than 2 in $r a d 53^{K 227 A}$ and $m r c 1 \Delta$ mutants. Among the 829 entries in oriDB, we defined 521 as active origins (with EdU signal in WT or checkpoint mutants rad53 $3^{K 227 A}$ and $m r c 1 \Delta$ ), in which 256 was categorized as early origins (with EdU signal in WT) and 265 as late origins (with signal in checkpoint mutants but not in WT). The remaining 308 entries do not have significant signal under our condition and were deemed inactive origins.

\section{Computational analysis of sequence data}

540 The sequenced reads were trimmed by cutadapt with an option of "nextseq-trim", then aligned by 541 STAR ((Dobin et al., 2013)) in a paired-end mode to the sacCer3 genome masked at repetitive regions. The gene structure is referred from SGD reference genome annotation R64.1.1 as of Oct. 2018. For RNA-seq quantification analysis, the total counts of aligned reads were computed for each gene by applying "GeneCounts" mode. For ChIP-seq quantification analysis, the reads were mapped using the same pipeline. Additionally, peak calling was done by MACS2 in a narrow peak mode.

\section{Gene expression analysis}

Differentially expressed genes (DEGs) and their p-values were computed for each pair of the cases by nbinomWaldTest after size factor normalization using DESeq2 ((Love et al., 2014)). Using the list of DEGs, GO and KEGG enrichment analyses were performed via Pathview library. ClusterProfiler was applied to visualize fold changes of DEGs in each KEGG pathway. Co-expression analysis of significant DEGs was further performed base on co-expression network constructed in CoCoCoNet ((Lee et al., 2020)). CoCoCoNet has established the co-expression matrix of Spearman's correlation ranking based on 2,690 samples downloaded from SRA database. We carried out clustering for the correlation matrix downloaded from CoCoCoNet (yeast_metaAggnet) by dynamicTreeCut in R (or hierarchical clustering) to obtain at most 10 clusters. The enrichment analysis for the gene set of each cluster was performed in the same way with RNA-seq analysis.

560 For ChIP-seq signal normalization, two different methods were applied to different types of analysis.

561 For ChIP-seq residual analysis, we used simple normalization. In this process, each case sample is compared with the corresponding control sample of DNA input to compute log2 fold changes within each $25 \mathrm{bp}$ window reciprocally scaled by multiplying the total read counts of another sample. Then, the average of fold changes is computed for each duplicate. For ChIP-seq heatmap analysis, we employed the origin-aware normalization to account for the higher background around origin region as a result of DNA replication. In the origin-aware normalization, the same computation used in simple normalization, or $\log 2$ fold change with scaling by the total read count, is independently applied for the region proximal to the origins and others. For the heatmap presented in this paper, the origin-proximal region is defined as the region within 5,000 bp upstream and downstream.

\section{Heatmap analyses at origins and TSS}

After the average fold change computation and normalization from ChIP-seq signals, the signal strength is visualized around the target regions such as TSSs and replication origins are extracted using normalizeToMatrix function in EnrichedHeatmap (window size is $25 \mathrm{bp}$ and average mode is

$575 \mathrm{w} 0$ ). We ordered heatmaps to examine a different signal enrichment pattern for the characteristics of 
each origin or gene. For the heatmap row of each origin is ordered by the assigned replication timing for ChIP-seq signals around replication origins. The replication time for the origins are annotated with the replication timing data published previously ((Yabuki et al., 2002)). From the estimated replication time for each 1,000 bp window, we extracted the closest window from the center of each replication origin and assigned it as the representative replication timing if their distance is no more than 5,000 bp. Early and late origins groups are categorized according to the definition of the origintypes using the replication profile data from this study. The final set of the replication origins used in the heatmap analysis are obtained after filtering out the replication origins overlapped with any of 238 hyper-ChIPable regions defined in the previous study ((Teytelman et al., 2013)). In total, 167 early and 231 late origins pass this filter and are used in the heatmaps analysis in this study. For heatmaps of the ChIP-seq signals around TSS, we ordered genes based on RNA-seq fold changes for all DEGs or per co-expression cluster of DEGs based on gene co-expression network constructed in CoCoCoNet ((Lee et al., 2020)).

\section{ChIP-seq residual analysis}

To detect the time-dependent increase or decrease of Rad53 binding signals, we first focused on the $500 \mathrm{bp}$ window upstream from each TSS and computed the sum of the fold change signals estimated for each 25-bp window scaled by the window size as an activity of Rad53 binding for each gene. The overall activity scores are varied for each time point probably because of the different Rad53 protein level or other batch-specific reasons. To adjust such sample specific differences for a fair comparison, a linear regression is applied for the activity scores of all genes between G1 and other time points HU45 and HU90 using $1 \mathrm{~m}$ function in R. Then we selected top genes showing the deviated signals from the overall tendency according to the absolute residual values between the actual and predicted values, excluding the genes with signal value lower than -0.075 after scaling the maximal signal to 1 . Top 1,000 genes with the highest absolute residual values were selected from 2 sets of experiments. The common 435 genes among the duplicates were selected for further analysis.

\section{Data Availability}

All data supporting this work are available at public data sites. XXXX Source data are provide with this paper. XXXXURL.

\section{Code Availability}

$\mathrm{R}$ scripts for the co-expression analyses including clustering and enrichment analysis are available at https://github.com/carushi/yeast_coexp_analysis.

\section{Figure legends}

\section{Figure 1. DNA synthesis under stress caused by depletion of dNTP pool and during recovery from the stress.}

a, Yeast cells were synchronized in G1-phase and released into YPD containing $0.2 \mathrm{M}$ HU for $90 \mathrm{~min}$. (Top panel) Replication profiles of Chromosome IV for the wild type (WT), rad53 $3^{K 227 A}$ and $m r c 1 \Delta$ mutants. (Bottom panel) Replication profiles of wild type (WT), rad9 $\Delta$ and $\mathrm{rad} 53^{K 227 A}$ mutants. Red arrows point out some late origins. Ori-DB track indicate positions of replication origins annotated in OriDB (Siow et al., 2012). b, Scatter plot of EdU signals from early (E), late (L) and inactive (I) origins in $W T$ rad5 $3^{K 227 A}$ and $m r c 1 \Delta$ mutants. $\mathbf{c}$, Scheme for accessing DNA synthesis during 
recovery from HU stress. d, Cells that had progressed from G1 into S-phase in HU for 45 min. were released from the HU block and DNA synthesis labeled with EdU during an additional 25 min $(\mathrm{HU} \rightarrow \mathrm{S} 25$ ) or were continued in HU for another $45 \mathrm{~min}$. and labeled with EdU (HU $\rightarrow \mathrm{HU} 45)$. Replication profiles of Chromosome III is shown as an example. Yeast cells were synchronized in G1 phase and released into YPD containing $0.2 \mathrm{M} \mathrm{HU}$ for 45 and 90 min (HU45 and HU90, respectively). a, Replication profiles of Chromosome IV for the wild type (WT) and $m r c 1 \Delta$ mutants and for the WT and $r a d 53^{K 227 A}$ mutants. b, Replication profiles of Chromosome III of $W T$, rad9 $\Delta$ and $\mathrm{rad} 53^{K 227 A}$ mutants at HU90. Red arrows point out some late origins. Location of some heterochromatin regions are also indicated (black text and arrows).

Figure 2. Replisome status and checkpoint signaling at replication origins under replication stress

Cells were synchronized in G1-phase and released into YPD containing 0.2 M HU for 45 and 90 min (HU45 and HU90, respectively). WT, rad53 ${ }^{K 227 A}$ and $m r c 1 \Delta$ mutant cells at stages of G1, HU45 and HU90 were collected and processed for ChIP-seq analysis for distribution of Cdc45, $\gamma$-H2A and Rad53 at genome locations. a, c and e, Coverage tracks of ChIP-seq signals generated from mapped reads using BamCoverage with normalization of $1 \mathrm{X}$ genome size. $\mathbf{b}, \mathbf{d}$ and $\mathbf{f}$, Heatmaps of ChIP-seq signasl across $30 \mathrm{~kb}$ interval centered on active origins. Early origins (top panels) and late origins (bottom panels) are ordered according to the associated replication timing data reported in a previous study ((Yabuki et al., 2002)). a, Distribution of Cdc45 ChIP-seq signal on chromosome III. b, Heatmaps of Cdc45 ChIP-seq signal around active origins. $\mathbf{c}, \gamma$-H2A ChIP-seq signal on chromosome III. d, Heatmaps of $\gamma$-H2A ChIP-seq signal around active origins. e, Rad53 ChIP-seq signal on chromosome III. f, Heatmaps of Rad53 ChIP-seq signal around active origins.

\section{Figure 2 - figure supplement 1. Heatmaps of ChIP-seq signal across $30 \mathrm{~kb}$ centered on all active origins} Cells were synchronized in G1 phase and released into YPD containing $0.2 \mathrm{M} \mathrm{HU}$ for 45 and $90 \mathrm{~min}$ (HU45 and HU90, respectively). WT, rad53 ${ }^{K 227 A}$ and $m r c 1 \Delta$ mutant cells at stages of G1, HU45 and HU90 were collected and processed for ChIP-seq analysis. a, b, and c, Heatmaps of ChIP-seq signal of $\mathrm{Cdc} 45, \gamma-\mathrm{H} 2 \mathrm{~A}$ and Rad53, respectively, across $30 \mathrm{~kb}$ centered on all active origins as defined in this study (see Definition of the origin-types in methods section). Origins are ordered according to the associated replication timing data reported in previous study (Yabuki et al., 2002). mrcl $\Delta$ mutant cells at stages of G1, HU45 and HU90. b, Rad53 ChIP-seq profiles in WT, sml1 ,

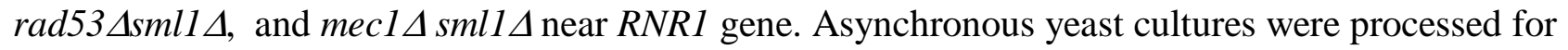
ChIP-seq analysis for distribution of Rad53. Tracks from WT G1, HU45 and HU90 are also included for reference. c, Heatmaps and average signals of Rad53 ChIP-seq signal across $2 \mathrm{~kb}$ interval

666 Figure 3 - figure supplement 1. Recruitment of Rad53 to upstream TSS depends on the 667 presence of Rad53 
a. Rad53 ChIP-seq profiles in $W T, \operatorname{sml1} \Delta, \operatorname{rad53} \Delta \operatorname{sml1} \Delta$, and mec1 $\Delta \operatorname{sml} 1 \Delta$ for chromosome III. Asynchronous yeast cultures were processed for ChIP-seq analysis for distribution of Rad53. The results from two independent experiments are shown. Experiment 1 compares only sml1 $\Delta$, rad53 $\Delta s m l 1 \Delta$. Experiment 2 is the same shown in Figure 3b. Tracks from WT G1, HU45 and HU90 are also included for reference. $\mathbf{b}$, Pie charts showing the distribution of Rad53 ChIP-seq peaks in relation to genes.

Figure 3 - figure supplement 2. Relative level of Rad53 protein changes in cells a, Immunoblots monitoring protein status for Rad53, Orc6, $\gamma-\mathrm{H} 2 \mathrm{~A}$ and Sml1 during checkpoint activation from G1 to HU45 and HU90. b, Comparison of Rad53 protein level in G1 extracts from $W T, \operatorname{rad} 53^{K 227 A}, m r c 1 \Delta$ and $\mathrm{rad} 9 \Delta$ cells. Blots for Orc6, $\gamma-\mathrm{H} 2 \mathrm{~A}$ and $\mathrm{Sml1}$ are included for reference. c, Comparison of Rad53 protein level in HU90 extracts from $W T$, rad53 ${ }^{K 227 A}$, mrcl $\Delta$ and $\mathrm{rad} 9 \Delta$ cells. $14 \%$ SDS-PAGE was used for the Rad53 blot to allow collapsing of all phosphorylated forms into a single band. Two-fold dilutions of the samples are loaded.

Figure 3 - figure supplement 3. Comparison of $\gamma \mathrm{H} 2 \mathrm{~A}$ enrichment with and without sequences enriched promiscuously in previous studies. Heatmaps surrounding the TSS (plus and minus 1000 bp) using ChIP-Seq data with antibodies against $-\gamma \mathrm{H} 2 \mathrm{~A}$. Panel a shows the complete data set and panel $b$ shows the data set following removal of the so-called hyper-ChIPable regions observed previously (Park et al., 2013; Teytelman et al., 2013).

Figure 3 - figure supplement 4. Comparison of Rad53 enrichment with and without sequences enriched promiscuously in previous studies. Heatmaps surrounding TSS (plus and minus $1000 \mathrm{bp}$ ) using ChIP-Seq data with antibodies against Rad53. Panel a shows the complete data set, panel b shows the data set following removal of the hyper-ChIPable regions (Teytelman et al., 2013) and panel c shows the signal data surrounding the TSS for genes only at the so-called hyper-ChIPable regions observed by Teytelman et al. and Park et al. The pattern of localization is both upstream and downstream of the TSSs, unlike that found for Rad53 ChIP-Seq surrounding TSSs.

Figure 3 - figure supplement 5. KEGG enrichment for the hyperChIPable regions. The nonspecific, promiscuous hyper-ChIPable regions observed by Teytelman et al. 2013 and Park et al. 2013 were re-analyzed by KEGG analysis, showing mostly tRNA and snoRNA genes. 296 sequences were analyzed, much smaller than the regions enriched at TSSs using Rad53 antibodies.

\section{Figure 4. Identification of genes with Rad53 binding changes at the promoters}

a. Examples of coverage tracks for selected genes show Rad53 signal changes at the indicated promoters from G1 to HU. (b) Scatter plots compare the signals in G1and HU45 at 500 bp intervals upstream of TSS for all genes in WT. Orange dots indicated the 1000 genes with highest binding changes (Top $1000 \mathrm{DB}$ ) and satisfying the filter of minimal signal of -0.075 (Maximal =1). The two plots represent WT data sets from two separate experiments (see text). (c) Binding changes for 435 genes that are in both sets of Top 1000 DB (435 Top DB overlap).

Figure 5. Gene expression changes in WT and checkpoint mutants under stress and the tendency of higher Rad53 binding at promoter of genes with significant differential expression a. Rank data analysis of RNA-seq samples. WT, $\operatorname{rad} 9 \Delta, \operatorname{rad} 53^{K 227 A}$ and $m r c 1 \Delta$ cells were synchronized in G1-phase and released into YPD containing 0.2 M HU for 45 and 90 min (HU45 and 
HU90, respectively). Cells at stages of G1, HU45 and HU90 were collected and processed for RNAseq analysis. (b) Bar graph summarizing the number of genes that show statistically significant differential expression (DEGs) in pair-wise comparison as indicated to the right. Blue bars, downregulated DEGs. Orange bars, up-regulated DEGs. (c) Average Rad53 ChIP-seq signal across $2 \mathrm{~kb}$ interval centered on at TSS for statistically significant (red) and insignificant (cyan) DEGs.

Figure 5 - figure supplement 1. Average Rad53 ChIP-seq signal and heatmaps of signal across $2 \mathrm{~kb}$ interval centered on TSS for various groups of time-dependent differentially expressed genes (DEGs) for DEGs in WT(G1 $\rightarrow$ HU45) and in WT(G1 $\rightarrow$ HU90). b and c, Rad53 ChIP-seq signal DEGs in $\operatorname{rad}^{2} 3^{K 227 A}(\mathrm{G} 1 \rightarrow \mathrm{HU} 45)$ and $m r c 1 \Delta(\mathrm{G} 1 \rightarrow \mathrm{HU} 45)$, respectively. Genes in each group are arranged according to the differential expression level from up to down. Average ChIP-seq signal for significant (red) and for insignificant (cyan) DEGs are plotted on top of the heatmap.

Figure 6. Correlation between differential binding of Rad53 at promoter and differential gene expression

a, Co-expression cluster matrix for significant DEGs in WT (G1 $\rightarrow$ HU45). Cluster (C): color codes for DEG clusters. Gene $(\mathrm{G})$ : level of differential expression. b, Heatmaps of Rad53 ChIP-seq signal across $2 \mathrm{~kb}$ interval centered on TSS for DEG clusters in a. Genes within each cluster are ordered by the level of expression changes from up to down. c, Scatter plots of binding changes against expression changes for the 236 significant DEGs in the 435 Top DB overlap group (top, left) and subgroups in clusters $1,2,4$ and 7.

Figure 6 - figure supplement 1. Rad53 ChIP-seq signal and heatmaps of signal across 2 kb interval centered on TSS for co-expression clusters in significant DEG in WT (G1 $\rightarrow$ HU45). Genes in each group are arranged according to the log2 based differential expression level from up to down. The three left most heatmap columns for WT samples are identical as in Figure 6b.

Figure 7. Origin-proximal DEGs are biased towards down-regulation in the mrc1 $\Delta$ mutant. a, Co-expression cluster matrix for significant DEGs in HU45 ( $m r c 1 \Delta$ vs WT). Cluster (C): color codes for DEG clusters. Gene $(\mathrm{G})$ : level of differential expression. b, Heatmaps of Rad53 ChIP-seq signal across $2 \mathrm{~kb}$ interval centered on TSS for DEG clusters in a. ChIP-seq signal in WT, rad53 ${ }^{K 227 A}$ and mrc1 $\Delta$ mutant cells at stages G1, HU45 and HU90 are shown. c, Summary of gene-origin relation in DEGs co-expression clusters for HU45 ( $m r c 1 \Delta$ vs WT). Distance between each TSS and its nearest origin center is indicated in pink gradient as well as light purple $(<2 \mathrm{~kb})$ and dark purple $(<1 \mathrm{~kb})$. Relative TSS-origin orientation and origin type are indicated. d, Proportion of down/up regulation of DEGs as categorized by TSS to origin distance (left panels), origin type within $5 \mathrm{~kb}$ (middle panels) and orientation (right panels; CD: co-directional; HO: head-on).

Figure 7 - figure supplement 1. Origin-proximal DEGs are biased towards down-regulation in the $\operatorname{rad53}{ }^{K 227 A}$ mutant.

a. Co-expression cluster matrix for significant DEGs in HU45 ( $r a d 53^{K 227 A}$ vs WT). Cluster (C): color codes for DEG clusters. Gene $(\mathrm{G})$ : level of differential expression. b, Heatmaps of Rad53 ChIP-seq signal across $2 \mathrm{~kb}$ interval centered on TSS for DEG clusters above. ChIP-seq signal in WT, rad53 ${ }^{K 227 A}$ and $m r c 1 \Delta$ mutant cells at stages G1, HU45 and HU90 are shown. c, Summary of gene- 
760 origin relation in DEGs co-expression clusters for HU45( rad53 $^{K 227 A}$ vs WT). Distance between each

761 TSS and its nearest origin center is indicated in pink gradient as well as light purple $(<2 \mathrm{~kb})$ and dark

762 purple $(<1 \mathrm{~kb})$. Relative TSS-origin orientation and origin type are indicated.

Figure 8. SBF plays a major role in the localization of Rad53 to the promoters of its target genes under replication stress Differential binding of Rad53 at promoters and differential expression of target genes of SBF, MBF, Msn4 and Ste12. a, Top panels: scatter plots of binding changes (DB residual) and expression changes ( $\log 2$ Fold change) for targets of indicated transcription regulators that are in the 236 significant DEGs in the Top DB overlap (Figure 4). Bottom panels: scatter plots above adding the expression change data from the checkpoint mutants. Enrichment of transcription regulator targets in the 236 Top DB DEGs. b, Top panels: Scatter plots illustrating the Rad53 signal upstream of TSS for all genes in $W T$, ixr $1 \Delta$, swi $4 \Delta$ and swi6 $\Delta$ mutants. SBF targets found in the 435 Top DB overlap are show as orange or red diamond and RNRI in red diamond. Bottom panels: Close-up for specific area from above panels. c, Distribution of Rad53 ChIP-seq signal near selected Top DB genes that are targets of SBF, MBF, Ixr1 or Rfx1 in WT, ixrl $\Delta$, swi4 $\Delta$ and swi6 $\Delta$ mutants at stages of G1, HU45 and HU90.

\section{Figure 8 - figure supplement 1. Differential binding of Rad53 at promoter and differential} expression of target genes of SBF, MBF, Msn4 and Ste12. a, b, c and d, Targets of SBF, MBF, Ste12 and Msn4, respectively. Scatter plots of binding changes (DB residual) and expression changes ( $\log 2$ Fold change) for targets of indicated transcription regulators that are in the 236 significant DEGs in the Top DB overlap. Expression change data from $W T$, rad53 ${ }^{K 227 A}$, $m r c 1 \Delta$ and rad9 $\Delta$ are presented in pairwise manner for comparison and column 


\section{References}

Alepuz PM, Jovanovic A, Reiser V, Ammerer G. 2001. Stress-Induced MAP Kinase Hog1 Is Part of Transcription Activation Complexes. Mol Cell 7:767-777. doi:10.1016/s1097-2765(01)00221-0

Bacal J, Carretero MM, Pardo B, Barthe A, Sharma S, Chabes A, Lengronne A, Pasero P. 2018. Mrc1 and $\operatorname{Rad} 9$ cooperate to regulate initiation and elongation of DNA replication in response to DNA damage. The EMBO journal e99319. doi:10.15252/embj.201899319

Bastos de Oliveira FM, Kim D, Cussiol JR, Das J, Jeong MC, Doerfler L, Schmidt KH, Yu H, Smolka MB. 2015. Phosphoproteomics Reveals Distinct Modes of Mec1/ATR Signaling during DNA Replication. Mol Cell 57:1124-1132. doi:10.1016/j.molcel.2015.01.043

Behrouzi R, Lu C, Currie M, Jih G, Iglesias N, Moazed D. 2016. Heterochromatin assembly by interrupted Sir3 bridges across neighboring nucleosomes. Elife 5:e17556. doi:10.7554/elife.17556

Bell SP, Labib K. 2016. Chromosome Duplication in Saccharomyces cerevisiae. Genetics 203:10271067. doi:10.1534/genetics.115.186452

Bermejo R, Capra T, Jossen R, Colosio A, Frattini C, Carotenuto W, Cocito A, Doksani Y, Klein H, 146:233-246. doi:10.1016/j.cell.2011.06.033

Breeden LL. 2003. Periodic Transcription: A Cycle within a Cycle. Curr Biol 13:R31-R38. doi:10.1016/s0960-9822(02)01386-6

Bruhn C, Ajazi A, Ferrari E, Lanz MC, Batrin R, Choudhary R, Walvekar A, Laxman S, Longhese MP, Fabre E, Smolka MB, Foiani M. 2020. The Rad53CHK1/CHK2-Spt21NPAT and Tel1ATM axes couple glucose tolerance to histone dosage and subtelomeric silencing. Nat Commun 11:4154. doi:10.1038/s41467-020-17961-4

Bruin RAM de, Kalashnikova TI, Chahwan C, McDonald WH, Wohlschlegel J, Yates J, Russell P, Wittenberg C. 2006. Constraining G1-Specific Transcription to Late G1 Phase: The MBFAssociated Corepressor Nrm1 Acts via Negative Feedback. Mol Cell 23:483-496. doi:10.1016/j.molcel.2006.06.025

Callegari AJ, Kelly TJ. 2006. UV irradiation induces a postreplication DNA damage checkpoint. Proc National Acad Sci 103:15877-15882. doi:10.1073/pnas.0607343103

Can G, Kauerhof AC, Macak D, Zegerman P. 2018. Helicase Subunit Cdc45 Targets the Checkpoint Kinase Rad53 to Both Replication Initiation and Elongation Complexes after Fork Stalling. Mol Cell 73. doi:10.1016/j.molcel.2018.11.025 
Cobb JA, Schleker T, Rojas V, Bjergbaek L, Tercero JA, Gasser SM. 2005. Replisome instability, fork collapse, and gross chromosomal rearrangements arise synergistically from Mec1 kinase and RecQ helicase mutations. Genes \& Development 19:3055-3069. doi:10.1101/gad.361805

Desany BA, Alcasabas AA, Bachant JB, Elledge SJ. 1998. Recovery from DNA replicational stress is the essential function of the S-phase checkpoint pathway. Gene Dev 12:1-15. doi:10.1101/gad.12.18.2956

Devbhandari S, Remus D. 2020. Rad53 limits CMG helicase uncoupling from DNA synthesis at replication forks. Nat Struct Mol Biol 27:461-471. doi:10.1038/s41594-020-0407-7

Dobin A, Davis CA, Schlesinger F, Drenkow J, Zaleski C, Jha S, Batut P, Chaisson M, Gingeras TR. 2013. STAR: ultrafast universal RNA-seq aligner. Bioinformatics 29:15-21. doi:10.1093/bioinformatics/bts635

Forey R, Poveda A, Sharma S, Barthe A, Padioleau I, Renard C, Lambert R, Skrzypczak M, Ginalski K, Lengronne A, Chabes A, Pardo B, Pasero P. 2020. Mec1 Is Activated at the Onset of Normal S Phase by Low-dNTP Pools Impeding DNA Replication. Molecular Cell. doi:10.1016/j.molcel.2020.02.021

Freese NH, Norris DC, Loraine AE. 2016. Integrated genome browser: visual analytics platform for genomics. Bioinformatics 32:2089-2095. doi:10.1093/bioinformatics/btw069

Gilbert CS, Green CM, Lowndes NF. 2001. Budding Yeast Rad9 Is an ATP-Dependent Rad53 Activating Machine. Mol Cell 8:129-136. doi:10.1016/s1097-2765(01)00267-2 Saccharomyces cerevisiae Yta7 Regulates Histone Gene Expression. Genetics 179:291-304. doi:10.1534/genetics.107.086520

Hoch NC, Chen ESW, Buckland R, Wang S-C, Fazio A, Hammet A, Pellicioli A, Chabes A, Tsai Mkinase. Molecular and Cellular Biology 33:3202-3213. doi:10.1128/mcb.00474-13

Holzen TM, Sclafani RA. 2010. Genetic interaction of RAD53 protein kinase with histones is important for DNA replication. Cell Cycle 9:4735-4747. doi:10.4161/cc.9.23.14091 Induce Transcription by Inhibition of the Crt1 Repressor. Cell 94:595-605. doi:10.1016/s00928674(00)81601-3 
855

Jaehnig EJ, Kuo D, Hombauer H, Ideker TG, Kolodner RD. 2013. Checkpoint kinases regulate a global network of transcription factors in response to DNA damage. Cell reports 4:174-188. doi:10.1016/j.celrep.2013.05.041

Kim K-Y, Truman AW, Levin DE. 2008. Yeast Mpk1 Mitogen-Activated Protein Kinase Activates Transcription through Swi4/Swi6 by a Noncatalytic Mechanism That Requires Upstream Signal? †. Mol Cell Biol 28:2579-2589. doi:10.1128/mcb.01795-07

Kumar S, Huberman JA. 2008. Checkpoint-Dependent Regulation of Origin Firing and Replication Fork Movement in Response to DNA Damage in Fission Yeast ?. Mol Cell Biol 29:602-611. doi:10.1128/mcb.01319-08

Langmead B. 2010. Aligning Short Sequencing Reads with Bowtie. Curr Protoc Bioinform 32:11.7.1-11.7.14. doi:10.1002/0471250953.bi1107s32

Lanz MC, Dibitetto D, Smolka MB. 2019. DNA damage kinase signaling: checkpoint and repair at 30 years. Embo J 38:e101801. doi:10.15252/embj.2019101801

Lao JP, Ulrich KM, Johnson JR, Newton BW, Vashisht AA, Wohlschlegel JA, Krogan NJ, Toczyski DP. 2018. The Yeast DNA Damage Checkpoint Kinase Rad53 Targets the Exoribonuclease, Xrn1. G3 Genes Genomes Genetics 8:g3.200767.2018. doi:10.1534/g3.118.200767

Lee J, Shah M, Ballouz S, Crow M, Gillis J. 2020. CoCoCoNet: conserved and comparative coexpression across a diverse set of species. Nucleic Acids Res 48:gkaa348-. doi:10.1093/nar/gkaa348

Lopes M, Cotta-Ramusino C, Pellicioli A, Liberi G, Plevani P, Muzi-Falconi M, Newlon CS, Foiani M. 2001. The DNA replication checkpoint response stabilizes stalled replication forks. Nature 412:557-561. doi:10.1038/35087613

Lou H, Komata M, Katou Y, Guan Z, Reis CC, Budd M, Shirahige K, Campbell JL. 2008. Mrc1 and DNA polymerase epsilon function together in linking DNA replication and the $\mathrm{S}$ phase checkpoint. Molecular Cell 32:106-117. doi:10.1016/j.molcel.2008.08.020

Love MI, Huber W, Anders S. 2014. Moderated estimation of fold change and dispersion for RNAseq data with DESeq2. Genome Biol 15:550. doi:10.1186/s13059-014-0550-8

Manfrini N, Gobbini E, Baldo V, Trovesi C, Lucchini G, Longhese MP. 2012. G1/S and G2/M Cyclin-Dependent Kinase Activities Commit Cells to Death in the Absence of the S-Phase Checkpoint. Mol Cell Biol 32:4971-4985. doi:10.1128/mcb.00956-12

McClure AW, Diffley J. 2021. Rad53 checkpoint kinase regulation of DNA replication fork rate via Mrc1 phosphorylation. doi:10.1101/2021.04.09.439171 
Oliveira FMB de, Harris MR, Brazauskas P, Bruin RAM de, Smolka MB. 2012. Linking DNA replication checkpoint to $\mathrm{MBF}$ cell-cycle transcription reveals a distinct class of G1/S genes. The EMBO Journal 31:1798-1810. doi:10.1038/emboj.2012.27

Osborn AJ, Elledge SJ. 2003. Mrc1 is a replication fork component whose phosphorylation in response to DNA replication stress activates Rad53. Gene Dev 17:1755-1767. doi:10.1101/gad.1098303

Pardo B, Crabbé L, Pasero P. 2017. Signaling pathways of replication stress in yeast. FEMS Yeast Research 17. doi:10.1093/femsyr/fow 101

Park D, Lee Y, Bhupindersingh G, Iyer VR. 2013. Widespread Misinterpretable ChIP-seq Bias in Yeast. Plos One 8:e83506. doi:10.1371/journal.pone.0083506

Paulovich AG, Hartwell LH. 1995. A checkpoint regulates the rate of progression through $\mathrm{S}$ phase in S. cerevisiae in Response to DNA damage. Cell 82:841-847. doi:10.1016/0092-8674(95)90481-6

Pellicioli A, Lucca C, Liberi G, Marini F, Lopes M, Plevani P, Romano A, Fiore PPD, Foiani M. 1999. Activation of Rad53 kinase in response to DNA damage and its effect in modulating phosphorylation of the lagging strand DNA polymerase. The EMBO Journal 18:6561-6572. doi:10.1093/emboj/18.22.6561

904

Ramírez F, Dündar F, Diehl S, Grüning BA, Manke T. 2014. deepTools: a flexible platform for exploring deep-sequencing data. Nucleic Acids Res 42:W187-W191. doi:10.1093/nar/gku365

Renard-Guillet C, Kanoh Y, Shirahige K, Masai H. 2014. Temporal and spatial regulation of eukaryotic DNA replication: From regulated initiation to genome-scale timing program. Semin Cell Dev Biol 30:110-120. doi:10.1016/j.semcdb.2014.04.014

Saldivar JC, Cortez D, Cimprich KA. 2017. The essential kinase ATR: ensuring faithful duplication of a challenging genome. Nature Publishing Group 16:1-15. doi:10.1038/nrm.2017.67

Sanz AB, García R, Rodríguez-Peña JM, Nombela C, Arroyo J. 2018. Slt2 MAPK association with chromatin is required for transcriptional activation of Rlm1 dependent genes upon cell wall stress. Biochimica Et Biophysica Acta Bba - Gene Regul Mech 1861:1029-1039. doi:10.1016/j.bbagrm.2018.09.005 both DNA Replication Initiation and Elongation: Single-Cell and -DNA Fiber Analyses?. Mol Cell Biol 27:5806-5818. doi:10.1128/mcb.02278-06 
920

921

922

923

924

925

926

927

928

929

930

931

932

933

934

935

936

937

938

939

940

941

942

943

944

945

946

947

948

949

950

951

952

953

954

Sheu Y-J, Kinney JB, Stillman B. 2016. Concerted activities of Mcm4, Sld3, and Dbf4 in control of origin activation and DNA replication fork progression. Genome Research 26:315-330. doi:10.1101/gr.195248.115

Sheu Y-J, Stillman B. 2006. Cdc7-Dbf4 phosphorylates MCM proteins via a docking site-mediated mechanism to promote S phase progression. Mol Cell 24:101-113. doi:10.1016/j.molcel.2006.07.033

Sidorova JM, Breeden LL. 2003. Rad53 Checkpoint Kinase Phosphorylation Site Preference Identified in the Swi6 Protein of Saccharomyces cerevisiae. Mol Cell Biol 23:3405-3416. doi:10.1128/mcb.23.10.3405-3416.2003

Siow CC, Nieduszynska SR, Müller CA, Nieduszynski CA. 2012. OriDB, the DNA replication origin database updated and extended. Nucleic Acids Res 40:D682-D686. doi:10.1093/nar/gkr1091

Smolka MB, Albuquerque CP, Chen S, Zhou H. 2007. Proteome-wide identification of in vivo targets of DNA damage checkpoint kinases. Proceedings of the National Academy of Sciences of the United States of America 104:10364-10369. doi:10.1073/pnas.0701622104

Smolka MB, Chen S, Maddox PS, Enserink JM, Albuquerque CP, Wei XX, Desai A, Kolodner RD, Zhou H. 2006. An FHA domain-mediated protein interaction network of Rad53 reveals its role in polarized cell growth. The Journal of Cell Biology 175:743-753. doi:10.1083/jcb.200605081

Szyjka SJ, Aparicio JG, Viggiani CJ, Knott S, Xu W, Tavaré S, Aparicio OM. 2008. Rad53 regulates replication fork restart after DNA damage in Saccharomyces cerevisiae. Gene Dev 22:1906-1920. doi:10.1101/gad.1660408

Tercero JA, Longhese MP, Diffley JFX. 2003. A Central Role for DNA Replication Forks in Checkpoint Activation and Response. Mol Cell 11:1323-1336. doi:10.1016/s10972765(03)00169-2

Teytelman L, Thurtle DM, Rine J, Oudenaarden A van. 2013. Highly expressed loci are vulnerable to misleading ChIP localization of multiple unrelated proteins. Proc National Acad Sci 110:1860218607. doi:10.1073/pnas.1316064110

Tourrière H, Versini G, Cordón-Preciado V, Alabert C, Pasero P. 2005. Mrc1 and Tof1 promote replication fork progression and recovery independently of Rad53. Molecular Cell 19:699-706.

Travesa A, Kuo D, Bruin RAM de, Kalashnikova TI, Guaderrama M, Thai K, Aslanian A, Smolka MB, Yates JR, Ideker T, Wittenberg C. 2012. DNA replication stress differentially regulates G1/S genes via Rad53-dependent inactivation of Nrm1. The EMBO Journal 31:1811-1822. doi:10.1038/emboj.2012.28

Tsaponina O, Barsoum E, Aström SU, Chabes A. 2011. Ixr1 is required for the expression of the ribonucleotide reductase Rnr1 and maintenance of dNTP pools. PLoS Genetics 7:e1002061. doi:10.1371/journal.pgen.1002061 
955 Viggiani CJ, Aparicio OM. 2006. New vectors for simplified construction of BrdU-Incorporating

956 strains of Saccharomyces cerevisiae. Yeast 23:1045-1051. doi:10.1002/yea.1406

957 Yabuki N, Terashima H, Kitada K. 2002. Mapping of early firing origins on a replication profile of

958 budding yeast. Genes Cells 7:781-789. doi:10.1046/j.1365-2443.2002.00559.x

959 Yeeles JTP, Janska A, Early A, Diffley JFX. 2016. How the Eukaryotic Replisome Achieves Rapid 960 and Efficient DNA Replication. Molecular Cell. doi:10.1016/j.molcel.2016.11.017

961 Zhao X, Georgieva B, Chabes A, Domkin V, Ippel JH, Schleucher J, Wijmenga S, Thelander L,

962 Rothstein R. 2000. Mutational and Structural Analyses of the Ribonucleotide Reductase Inhibitor 963 Sml1 Define Its Rnr1 Interaction Domain Whose Inactivation Allows Suppression of mec1 and 964 rad53 Lethality. Mol Cell Biol 20:9076-9083. doi:10.1128/mcb.20.23.9076-9083.2000 


\section{Supplemental Table 1}

968 Yeast strains used in this study

\begin{tabular}{|c|c|c|}
\hline Strain & genotype & source \\
\hline YS2571 & $\begin{array}{l}\text { MATa bar1 }:: \text { TRP1 URA3::BrdU-Inc ade2-1 can1-100 his3-11,- } \\
15 \text { leu2-3,112 trp1-1 ura3-1 }\end{array}$ & $\begin{array}{l}\text { Sheu et al } \\
2014 \text { (Sheu } \\
\text { et al., } \\
2014 \text { ) }\end{array}$ \\
\hline YS3110 & $\begin{array}{l}\text { MATa rad53 }{ }^{\text {K227A }}:: \text { KanMX4 bar1 } 1:: \text { TRP1 URA3::BrdU-Inc ade2-1 } \\
\text { can1-100 his3-11,-15 leu2-3,112 trp1-1 ura3-1 }\end{array}$ & $\begin{array}{l}\text { This } \\
\text { study }\end{array}$ \\
\hline YS3285 & $\begin{array}{l}\text { MATa mrc1 } \triangle:: \text { KanMX4 bar1 } 1:: \text { TRP1 URA3::BrdU-Inc ade2-1 } \\
\text { can1-100 his3-11,-15 leu2-3,112 trp1-1 ura3-1 }\end{array}$ & $\begin{array}{l}\text { This } \\
\text { study }\end{array}$ \\
\hline YS3382 & $\begin{array}{l}\text { MATa rad9 }:: \text { HIS3 barlA::TRP1 URA3::BrdU-Inc ade2-1 can1- } \\
100 \text { his3-11,-15 leu2-3,112 trp1-1 ura3-1 }\end{array}$ & $\begin{array}{l}\text { This } \\
\text { study }\end{array}$ \\
\hline YS3388 & $\begin{array}{l}\text { MATa ixr1 }:: H I S 3 \text { barl }:: \text { TRP1 URA3::BrdU-Inc ade2-1 can1- } \\
100 \text { his3-11,-15 leu2-3,112 trp1-1 ura3-1 }\end{array}$ & $\begin{array}{l}\text { This } \\
\text { study }\end{array}$ \\
\hline YS3401 & $\begin{array}{l}\text { MATa swi4A::HIS3 bar1A::TRP1 URA3::BrdU-Inc ade2-1 can1- } \\
100 \text { his3-11,-15 leu2-3,112 trp1-1 ura3-1 }\end{array}$ & $\begin{array}{l}\text { This } \\
\text { study }\end{array}$ \\
\hline YS3406 & $\begin{array}{l}\text { MATa swi64::HIS3 bar1 }:: \text { TRP1 URA3::BrdU-Inc ade2-1 can1- } \\
100 \text { his3-11,-15 leu2-3,112 trp1-1 ura3-1 }\end{array}$ & $\begin{array}{l}\text { This } \\
\text { study }\end{array}$ \\
\hline YS2828 & $\begin{array}{l}\text { MATa URA3::BrdU-Inc ade2-1 can1-100 his3-11,-15 leu2-3,112 } \\
\text { trp1-1 ura3-1 }\end{array}$ & $\begin{array}{l}\text { Sheu et al } \\
2016 \text { (Sheu } \\
\text { et al., } \\
2016 \text { ) }\end{array}$ \\
\hline YS3066 & $\begin{array}{l}\text { MATa sml1A::HIS3 URA3::BrdU-Inc ade2-1 can1-100 his3-11,-15 } \\
\text { leu2-3,112 trp1-1 ura3-1 }\end{array}$ & $\begin{array}{l}\text { Sheu et al } \\
2016 \text { (Sheu } \\
\text { et al., } \\
2016 \text { ) }\end{array}$ \\
\hline YS3075 & $\begin{array}{l}\text { MATa mec1 }:: \text { TRP1 sml14::HIS3 URA3::BrdU-Inc ade2-1 can1- } \\
100 \text { his3-11,-15 leu2-3,112 trp1-1 ura3-1 }\end{array}$ & $\begin{array}{l}\text { Sheu et al } \\
2016 \text { (Sheu } \\
\text { et al., } \\
2016 \text { ) }\end{array}$ \\
\hline YS3077 & 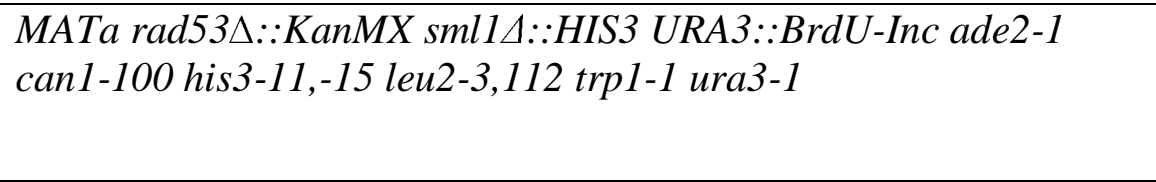 & $\begin{array}{l}\text { Sheu et al } \\
2016 \text { (Sheu } \\
\text { et al., } \\
2016 \text { ) }\end{array}$ \\
\hline
\end{tabular}



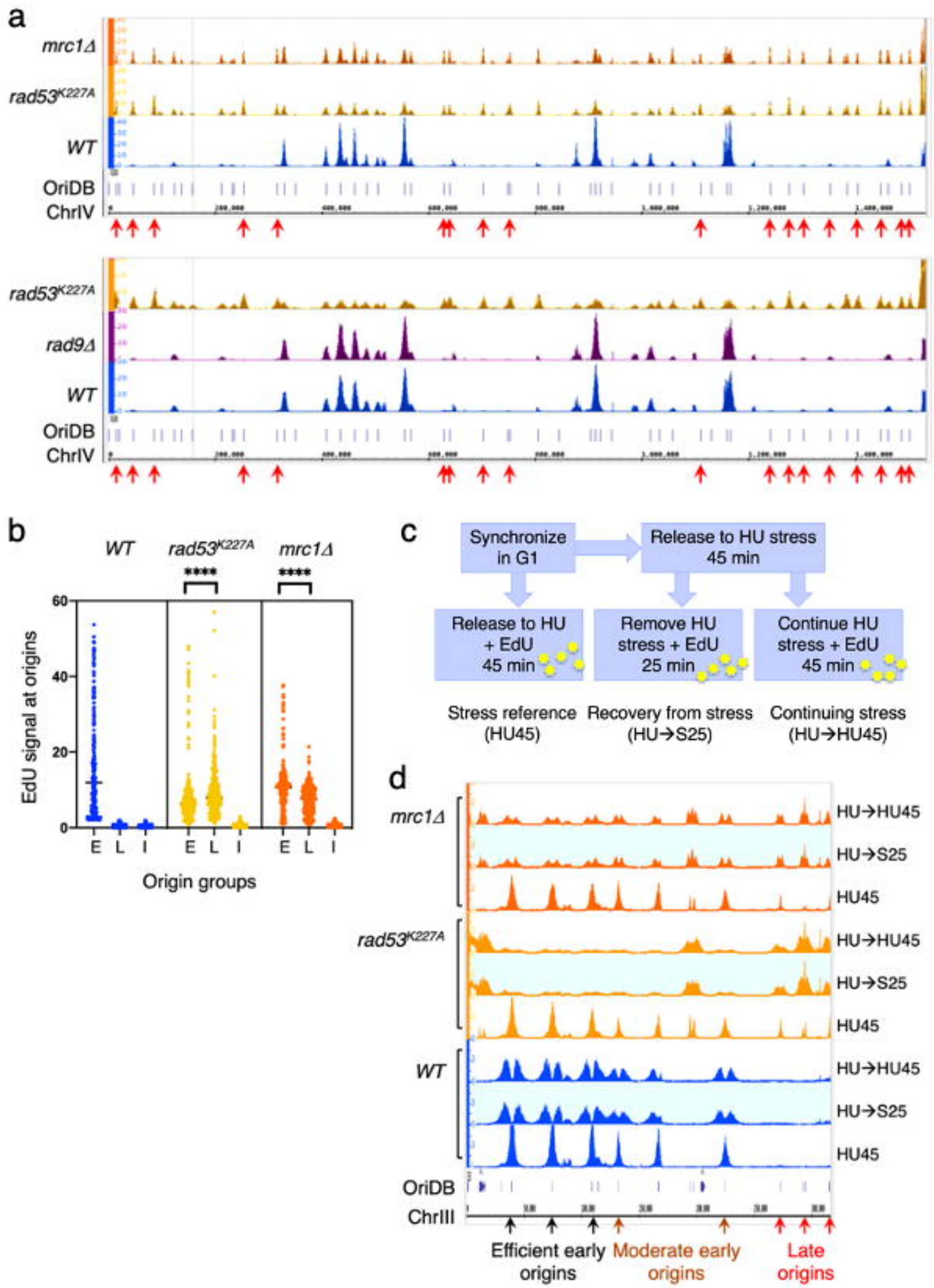

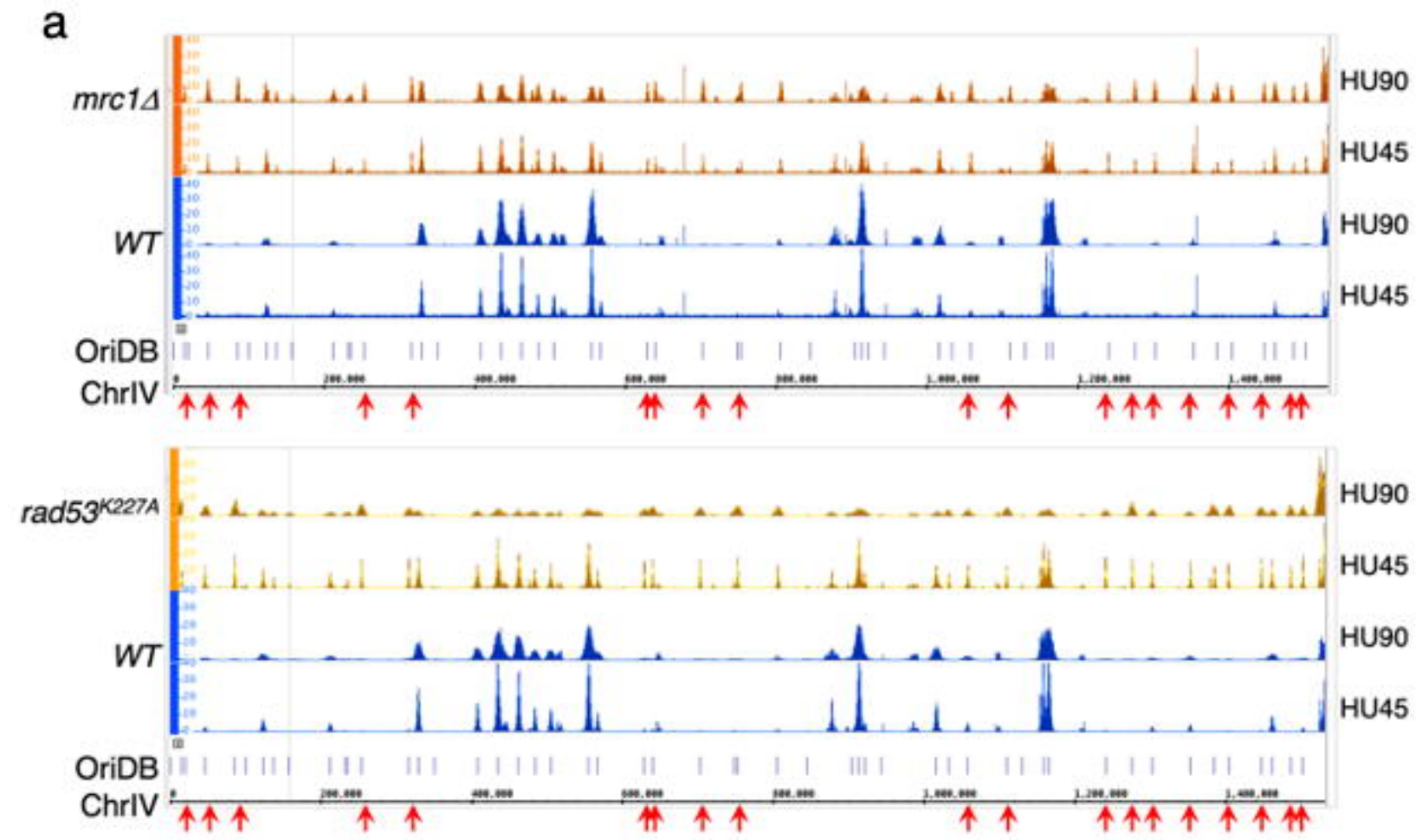

b

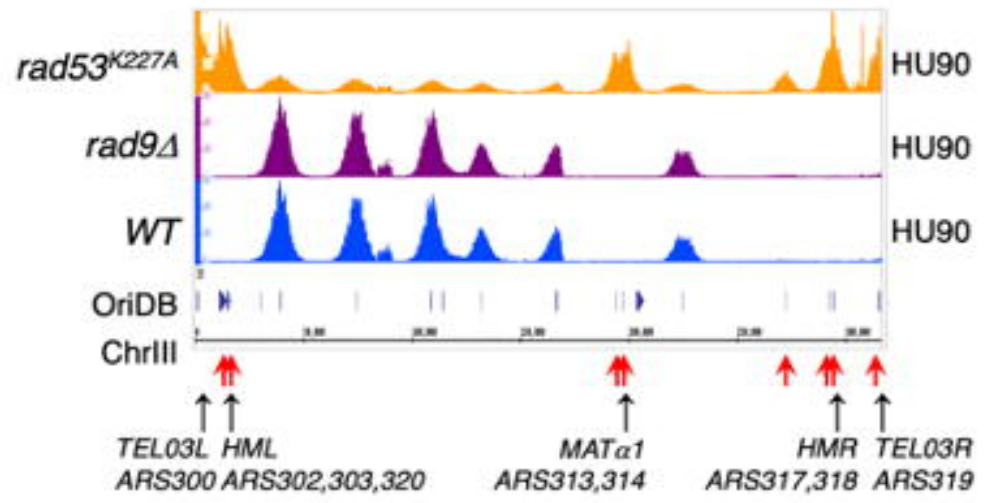



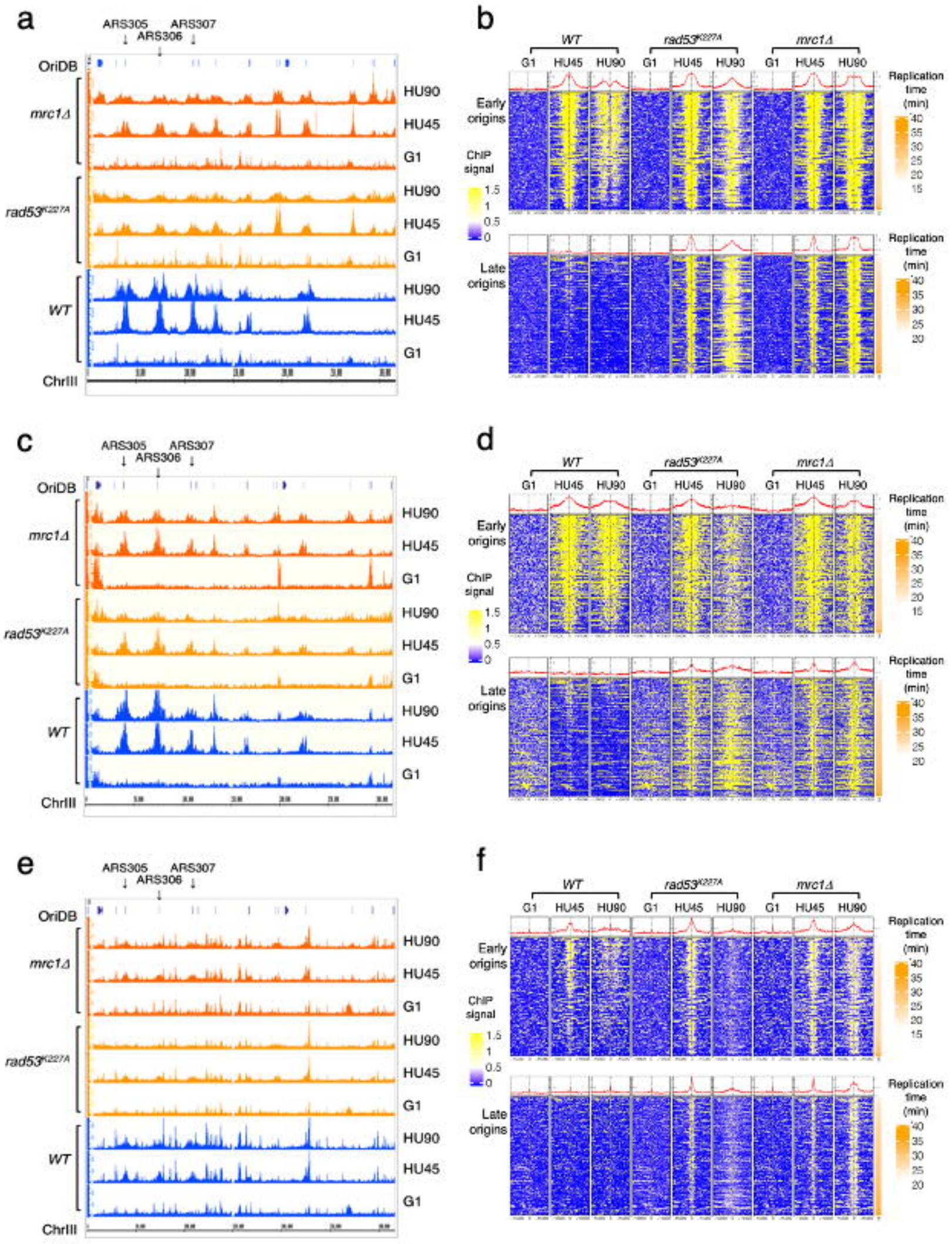
Figure 2 - figure supplement 1

a

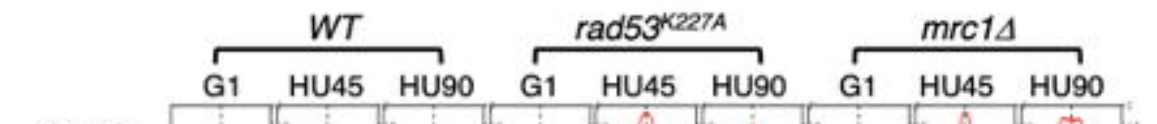

Cdc45
ChIP signal

$\begin{aligned} & 1.5 \\ & 1 \\ & 0.5 \\ = & 0\end{aligned}$
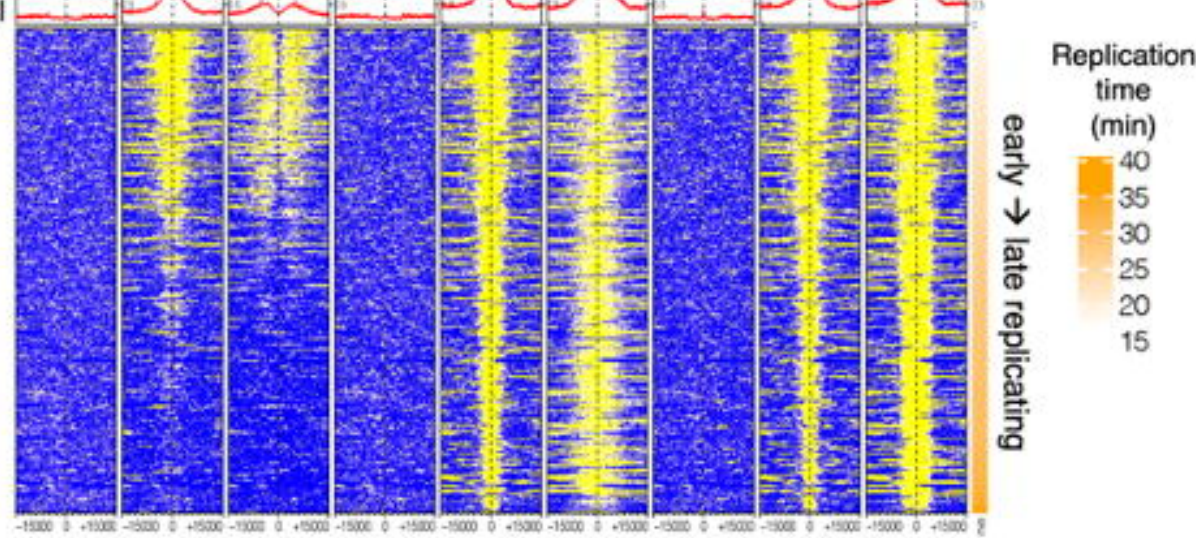

b

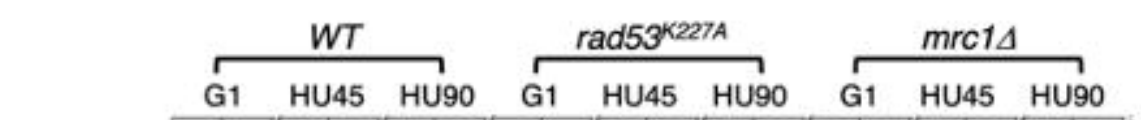
ChIP signal

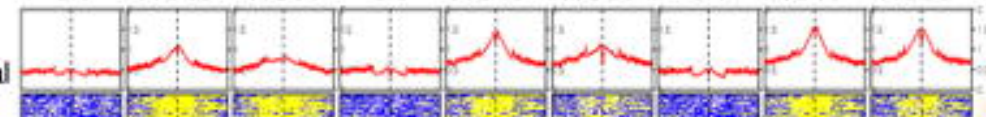

$\begin{aligned} & 1.5 \\ & 1 \\ & 0.5 \\ &= 0\end{aligned}$
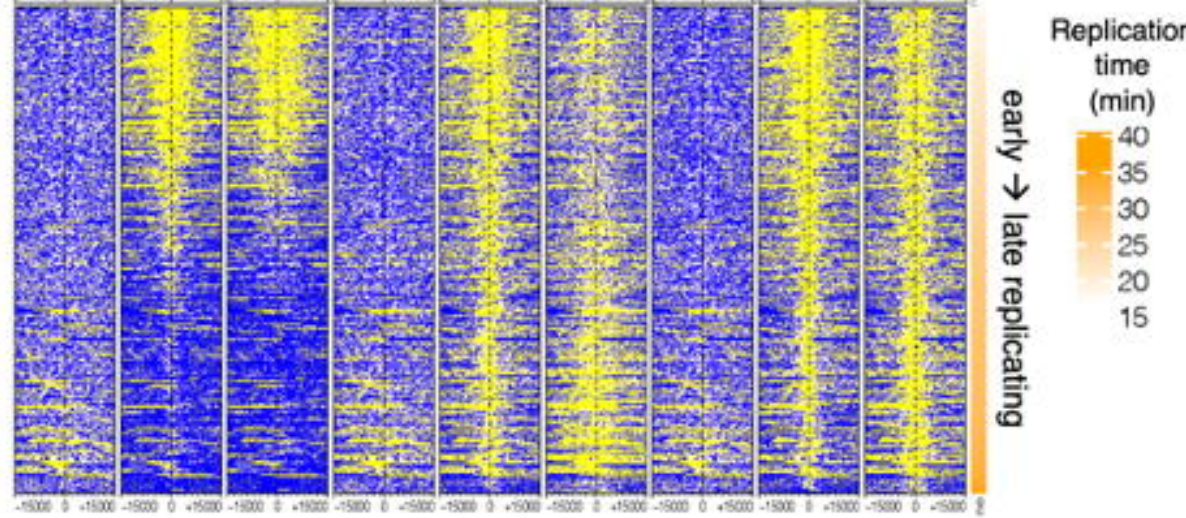

C
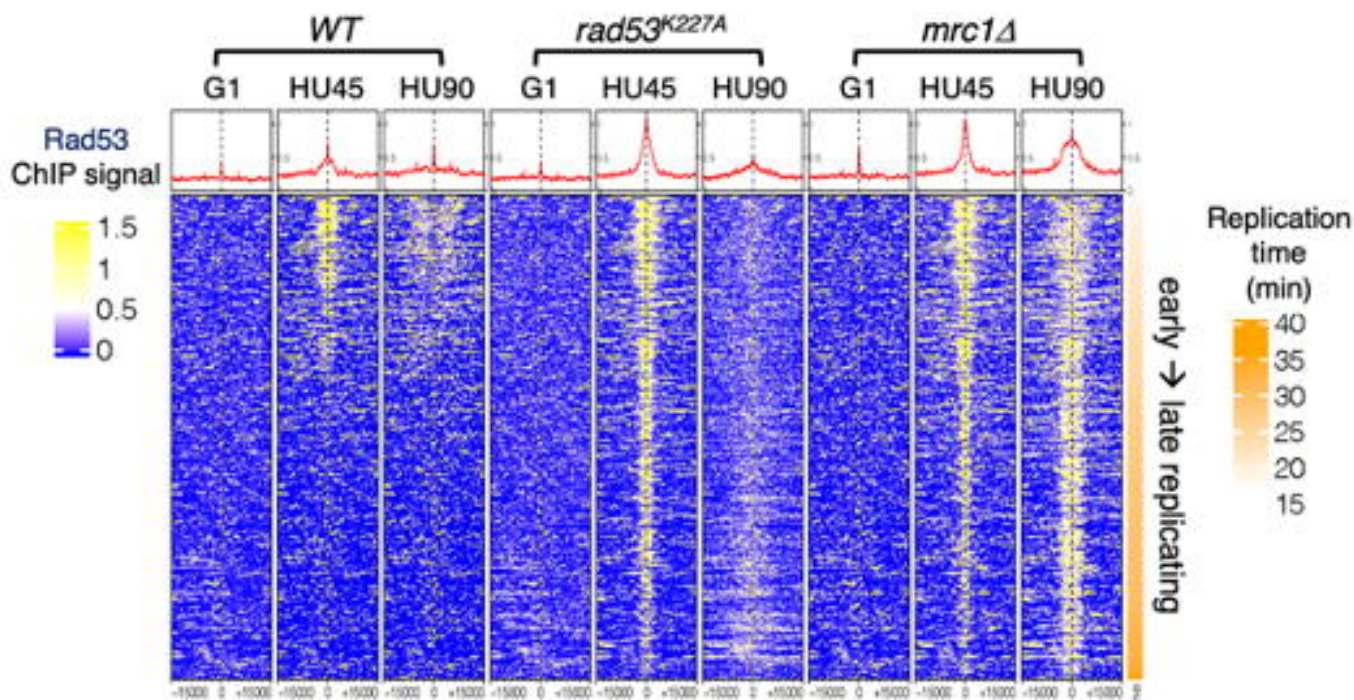
Figure 3
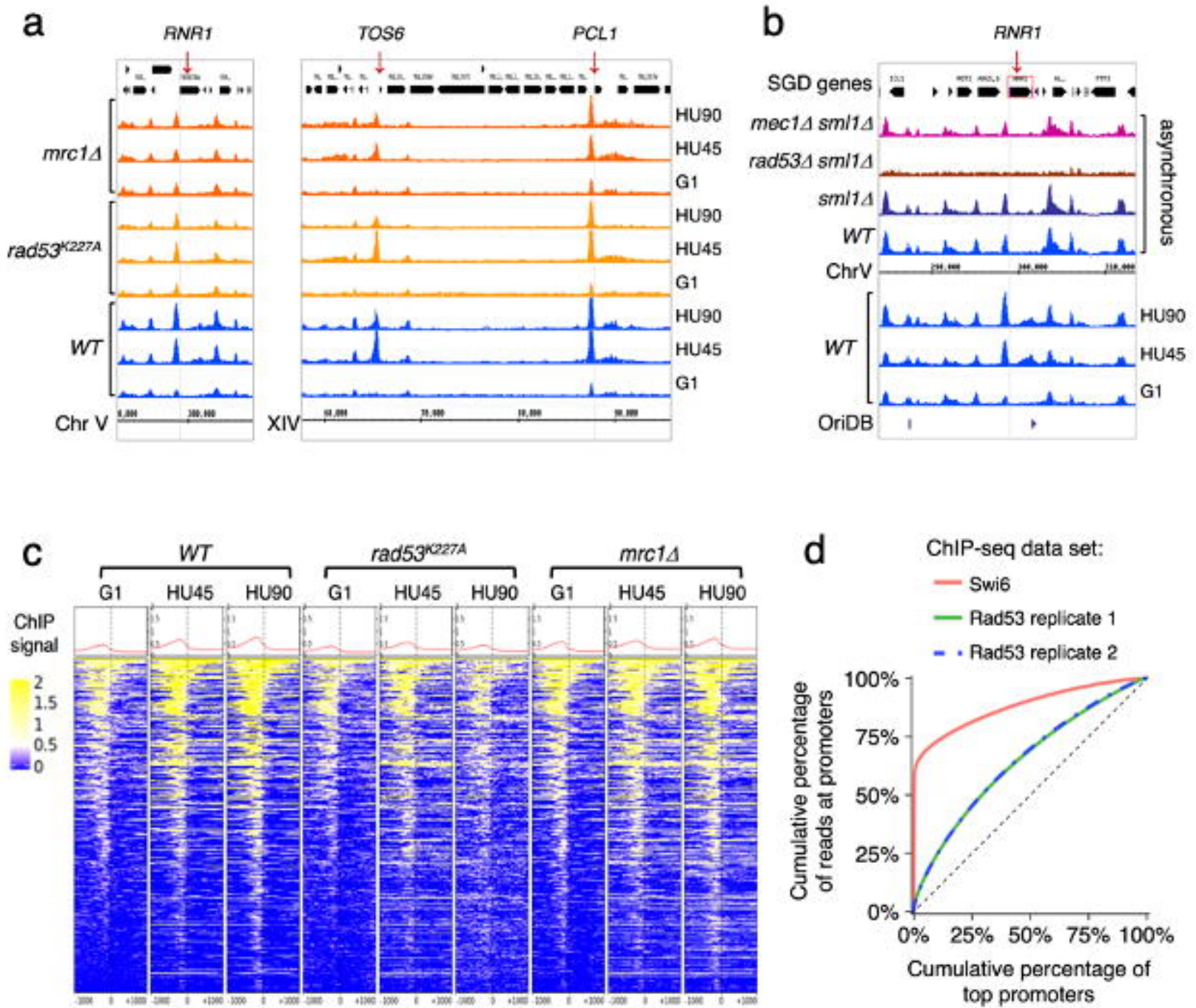
a

Rad53 ChIP-seq in WT and mutant cells

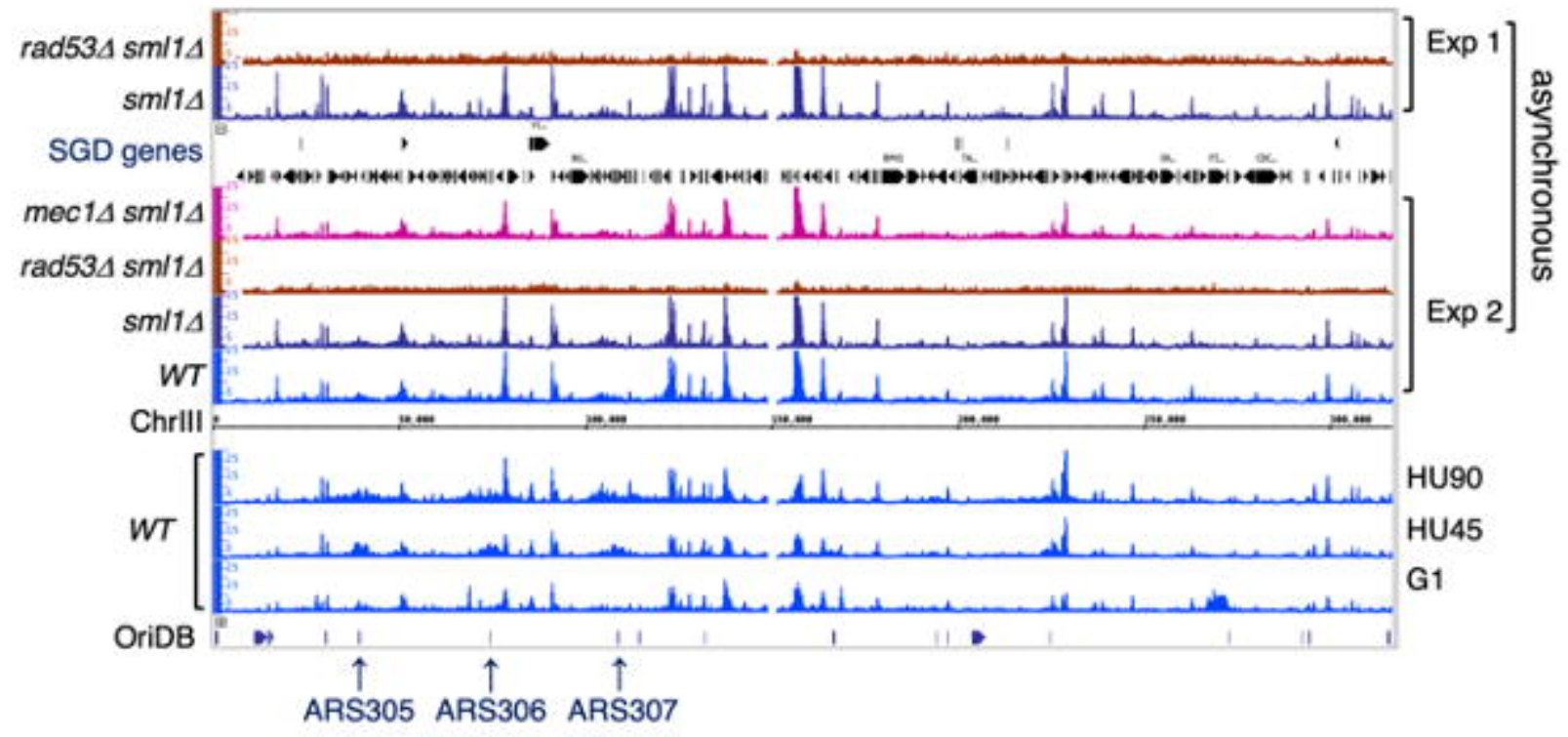

b Distribution of Rad53 peaks in relation to genes

G1

$(N=1372)$

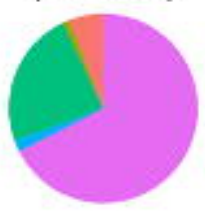

HU45

$(\mathrm{N}=1265)$

Feature Inside Peak $(5.8 \%)$
Overlap End $(1.2 \%)$
Overlap Start $(23.5 \%)$
Peak inside Feature $(2 \%)$
Upstream $(67.5 \%)$

Feature Inside Peak (5.8\%)

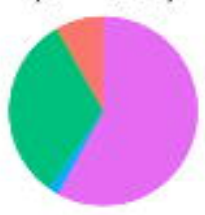

Feature Inside Peak ( $8 \%$ )

Overlap End $(0.4 \%)$

Overlap Start $(31,8 \%)$

Peak inside Feature (1.8\%)
HU90

$(N=1661)$

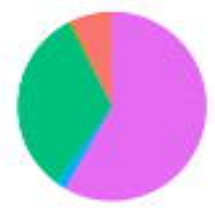

Upstream (58\%)
Common peaks

$(N=938)$

Feature Inside Peak (7.1\%)

Overlap End $(0.5 \%)$

Overlap Start (32.4\%)

Peak inside Feature (1.7\%)

Upstream (58.3\%)

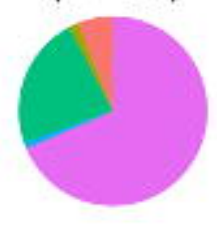

Feature Inside Peak (6.1\%)

Overlap End (1.6\%)

Overlap Start (22.7\%)

Peak inside Feature (1.1\%)

Upstream (68.6\%) 
Figure 3 - figure supplement 2

a

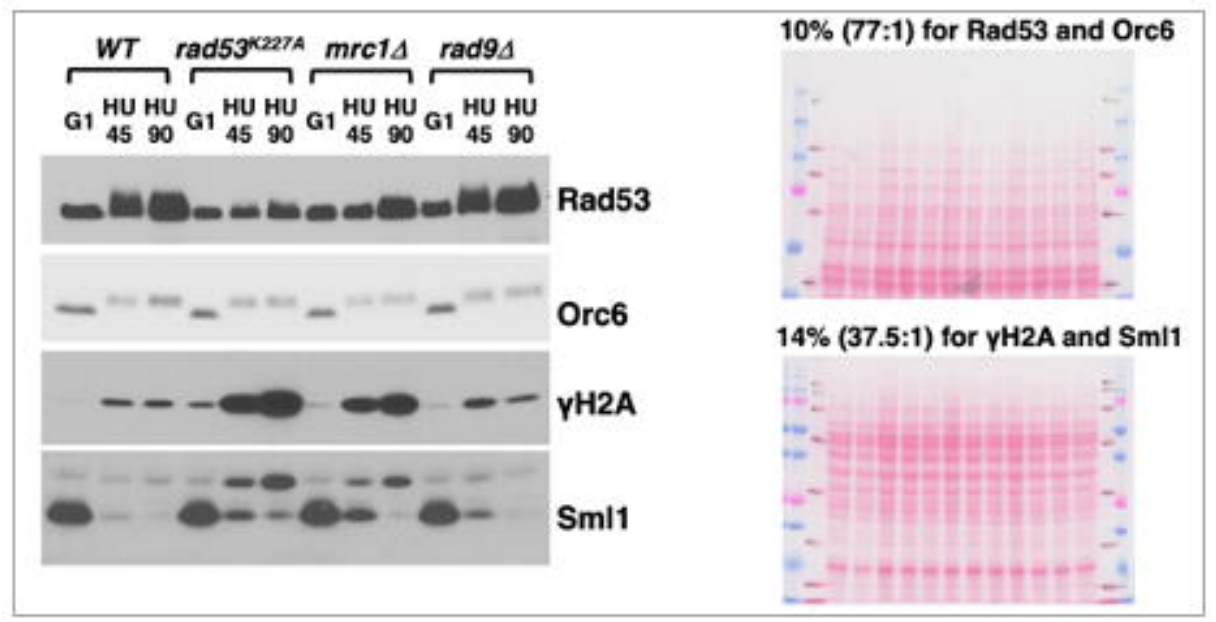

b

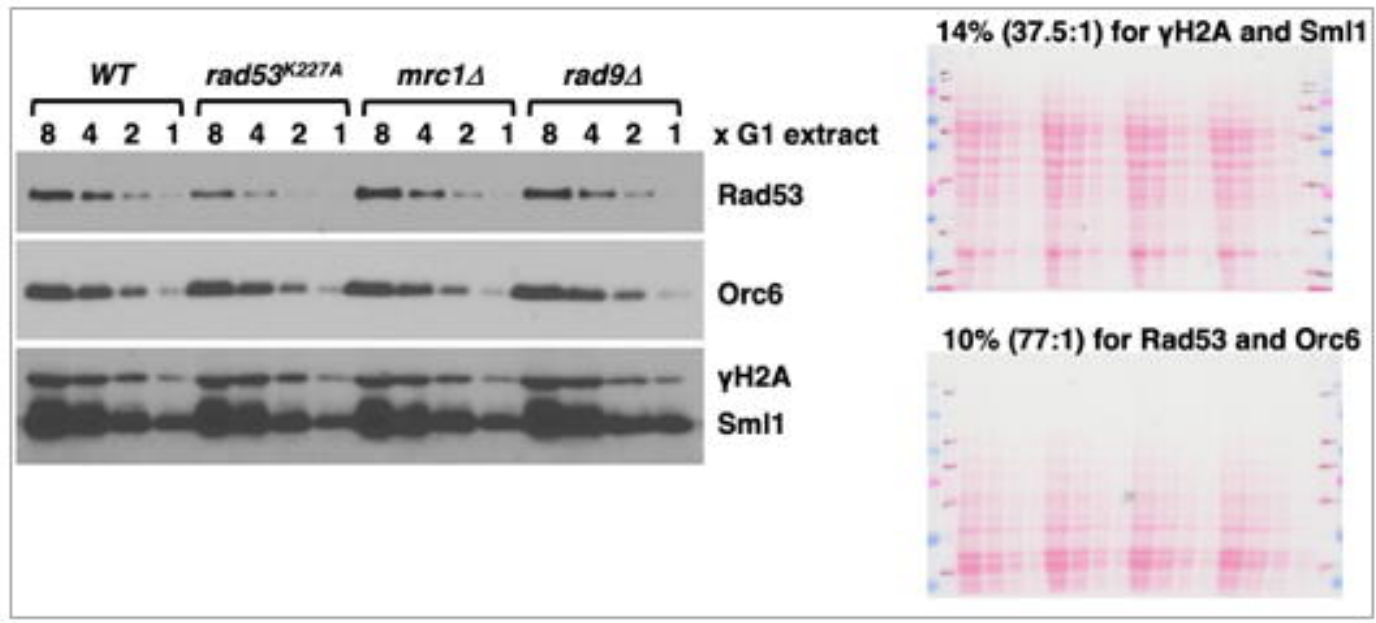

C

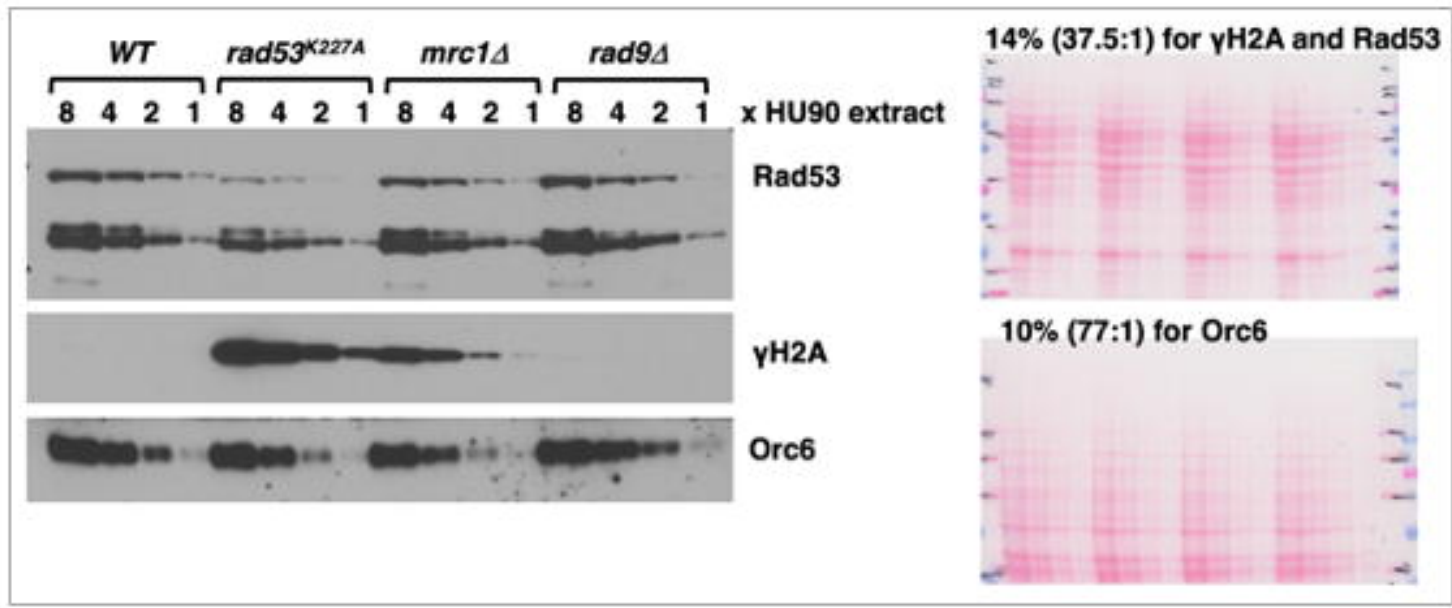


Figure 3 - figure supplement 3

a

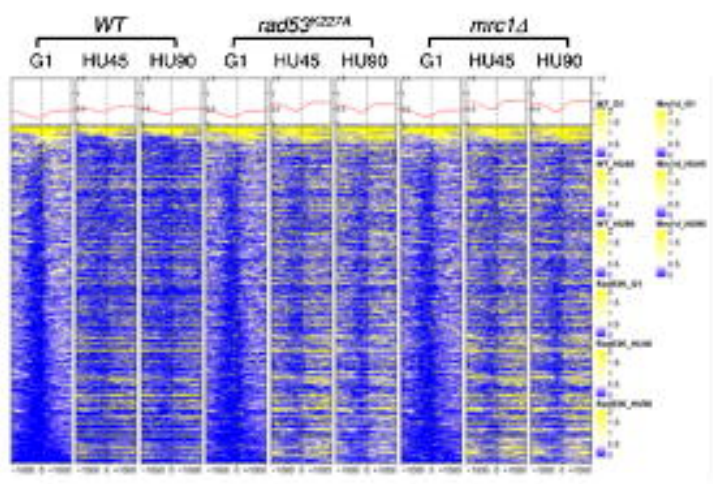

b

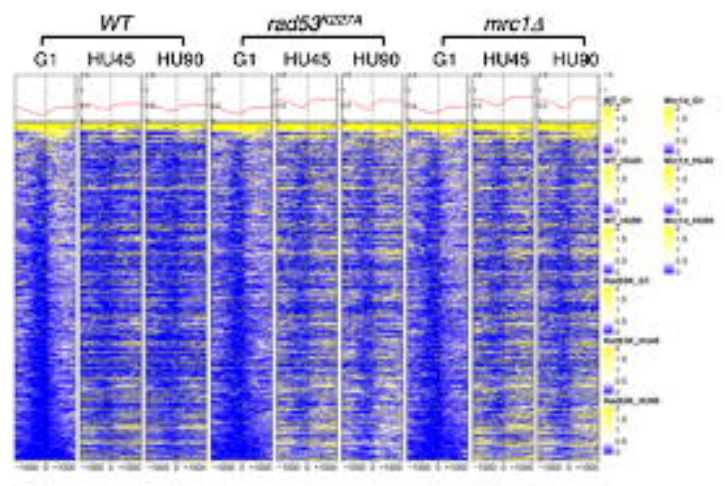


a

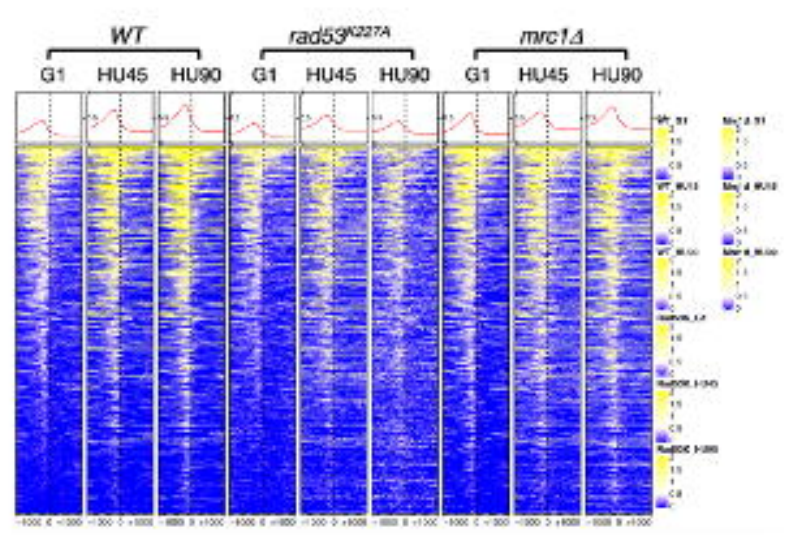

b

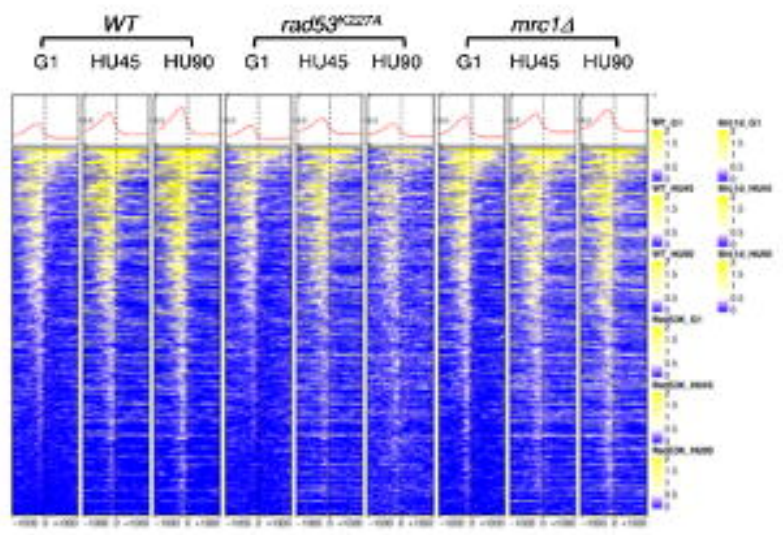

C

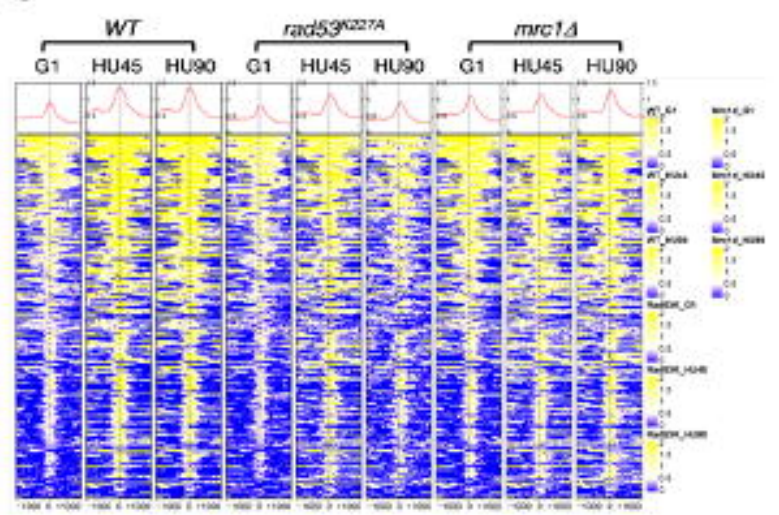



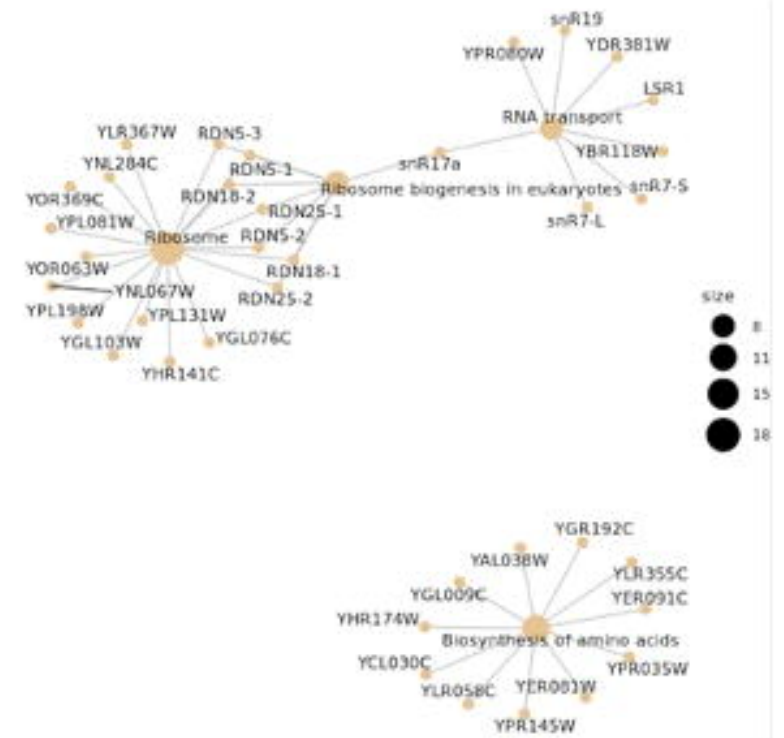

\section{Figure 3 - figure supplement 5}




\section{Figure 4}

a

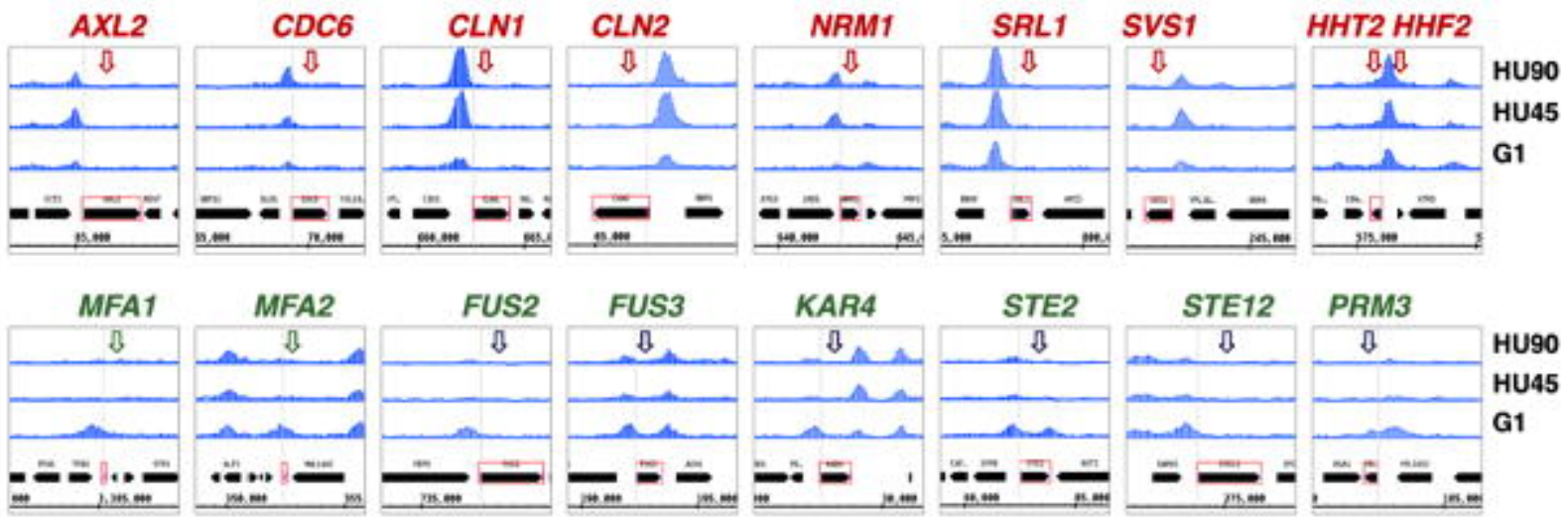

b

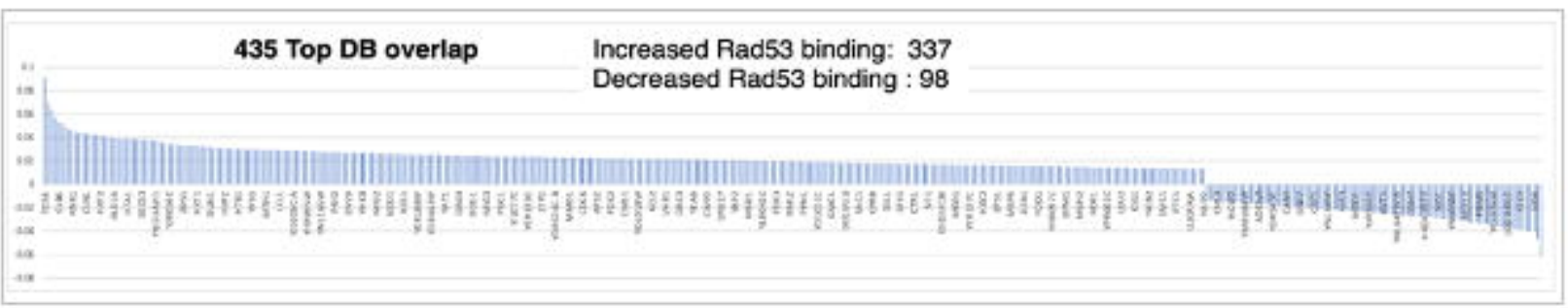

\section{C}

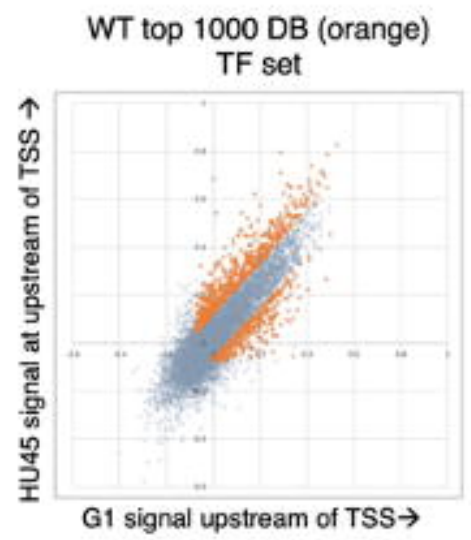

Increased Rad53 binding: 651 Decreased Rad53 binding: 349

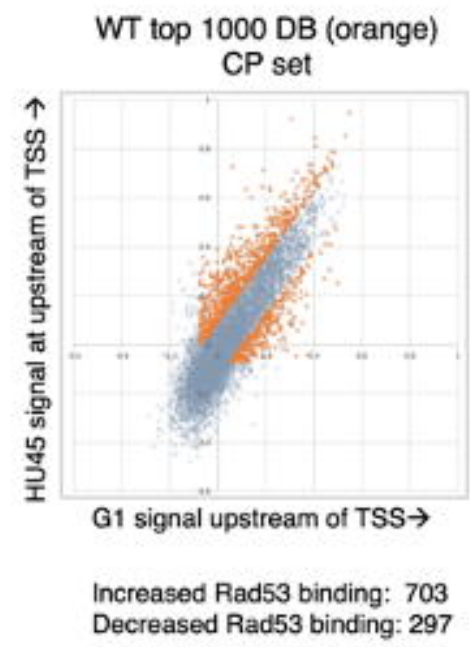

Increased Rad53 binding: 703
Decreased Rad53 binding: 297 
a
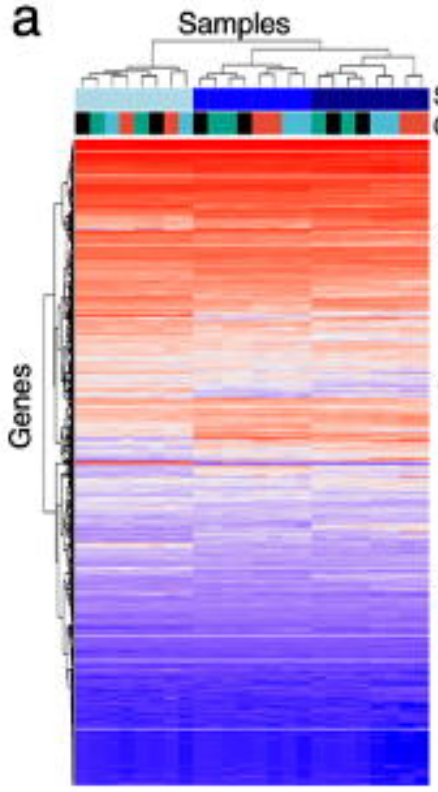

Stages

Genotypes

Stages:

G1

HU9O

Genotypes:

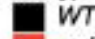

$\operatorname{rad} 53^{\circ} \approx 227 \mathrm{~A}$

mer 14

rad94

Ranking:

8000
6000
4000
2000
0 b

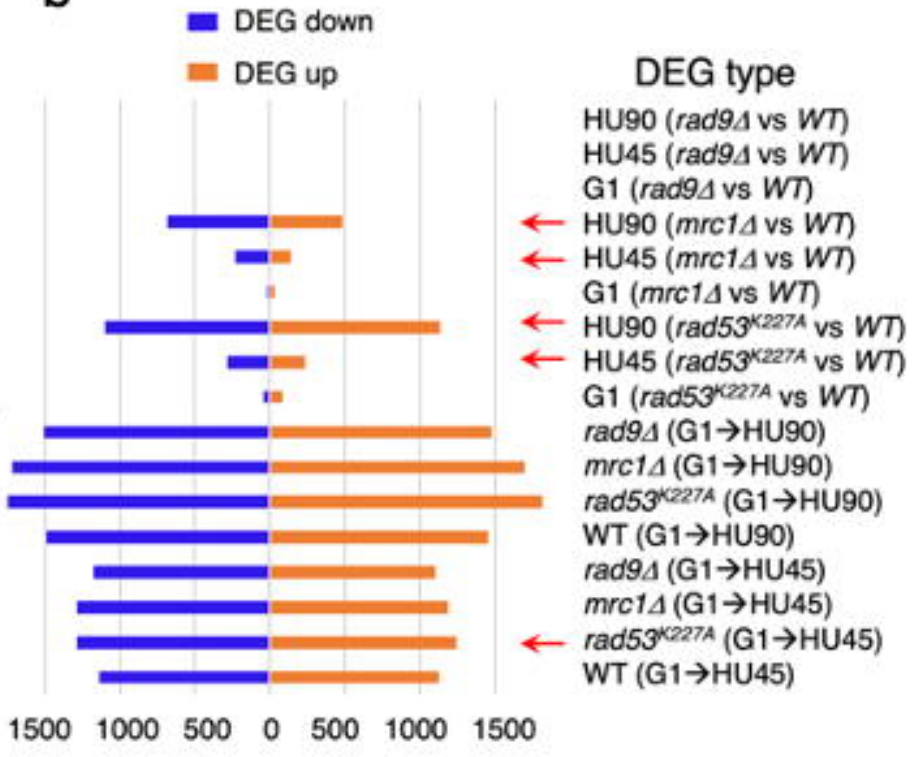

C

DEGs: WT (G1 $\rightarrow$ HU45) DEGs: WT (G1 $\rightarrow$ HU90)
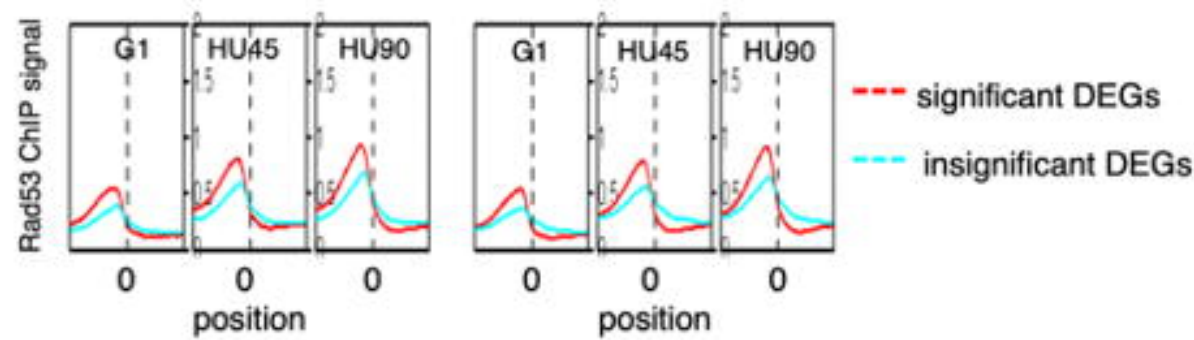


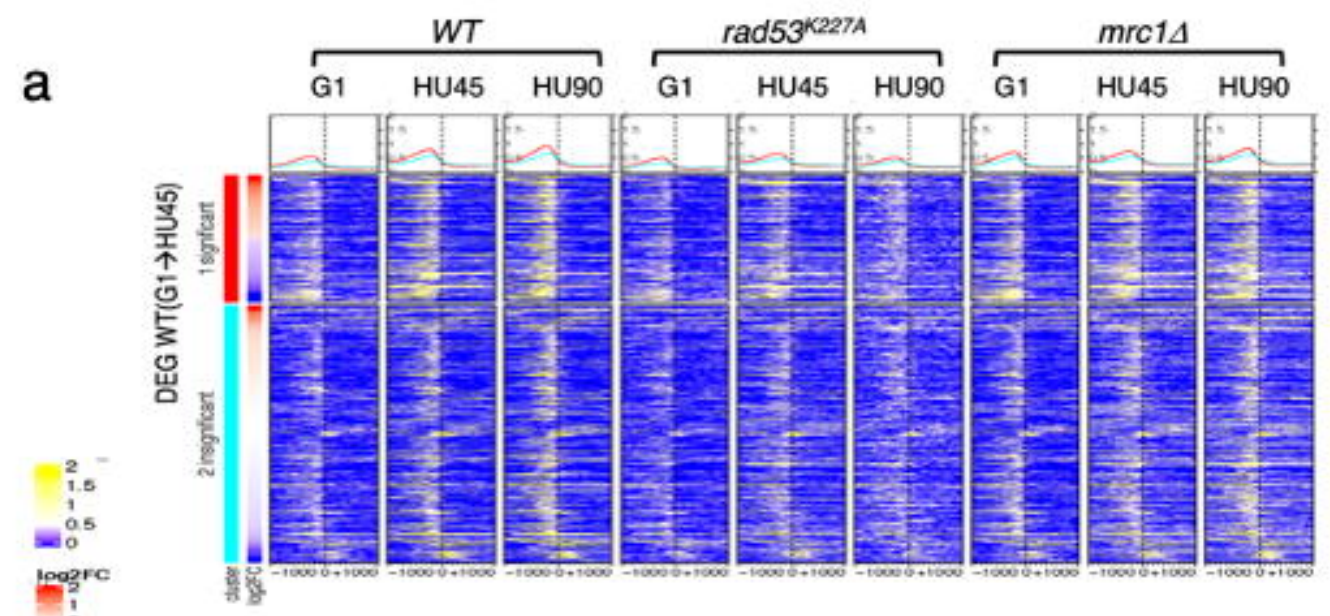

?

$\underbrace{0}_{-2}$

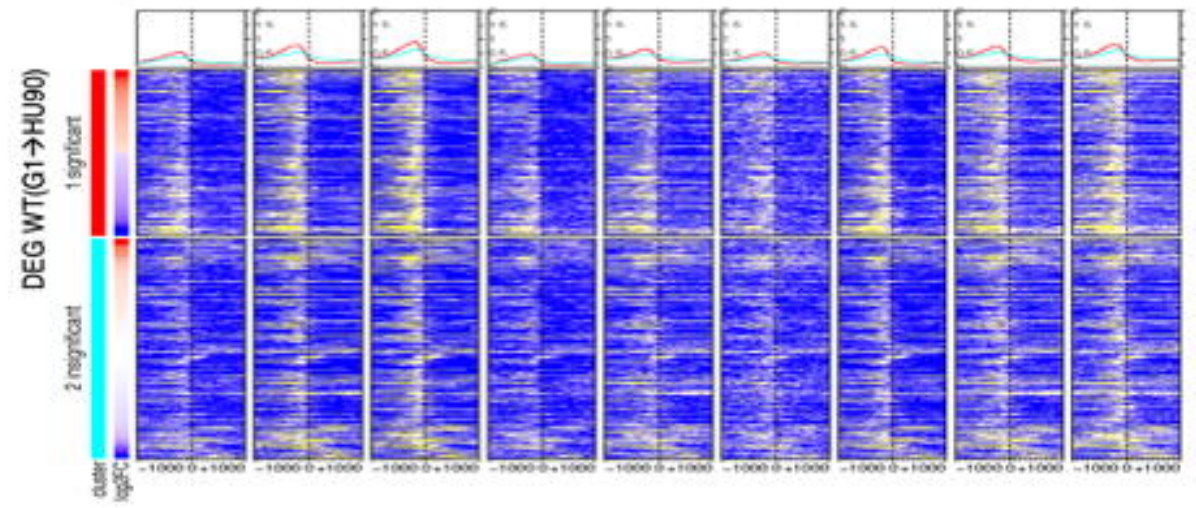

b
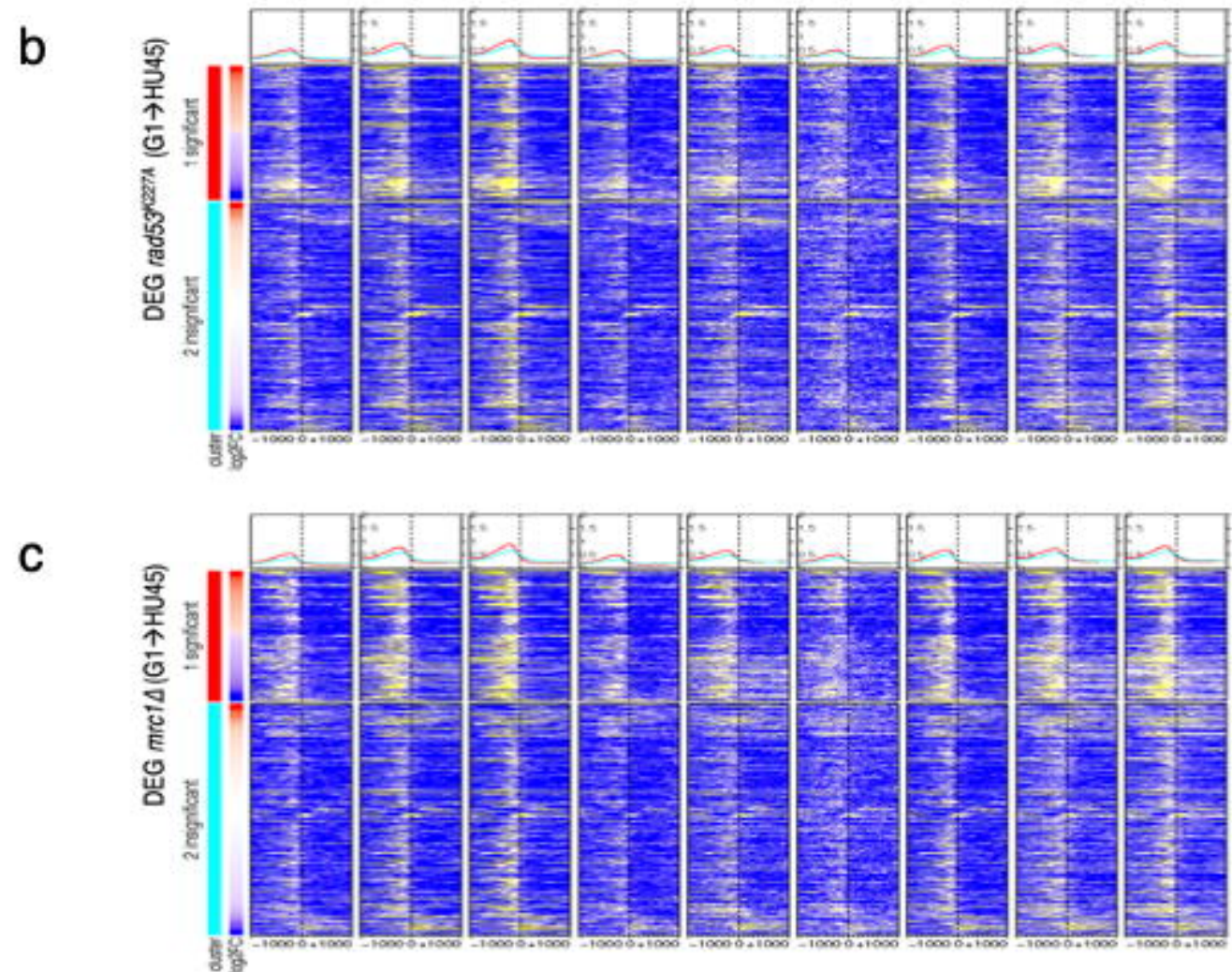
a

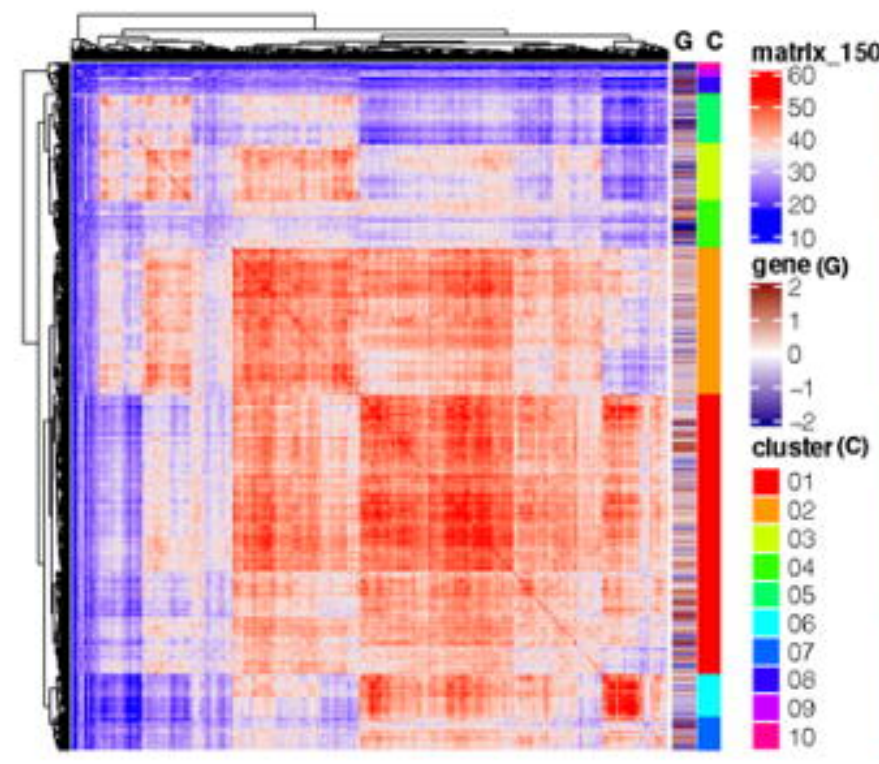

Summary of GO function DEG clusters for WT(G1 ->HU45)

\begin{tabular}{|l|}
\hline $\begin{array}{l}\text { 1. mitotic cell cycle, cell division } \\
\text { (up>down) }\end{array}$ \\
\hline 1. signaling, conjugation (down) \\
\hline $\begin{array}{l}\text { 2. ubiquitin-dependent protein } \\
\text { catabolic process, proteolysis (up) }\end{array}$ \\
\hline $\begin{array}{l}\text { 2. vesicle-mediated transport, } \\
\text { nucleotide transport (down) }\end{array}$ \\
\hline $\begin{array}{l}\text { 4. Processes related to mating } \\
\text { (down) }\end{array}$ \\
\hline $\begin{array}{l}\text { 5. oxidation-reduction process, } \\
\text { carbohydrate metabolism and energy } \\
\text { process }\end{array}$ \\
\hline 6. ribosome blogenesis \\
\hline $\begin{array}{l}\text { 7. Organic acid metabolism (down); } \\
\text { cell wall organization (up) }\end{array}$ \\
\hline
\end{tabular}
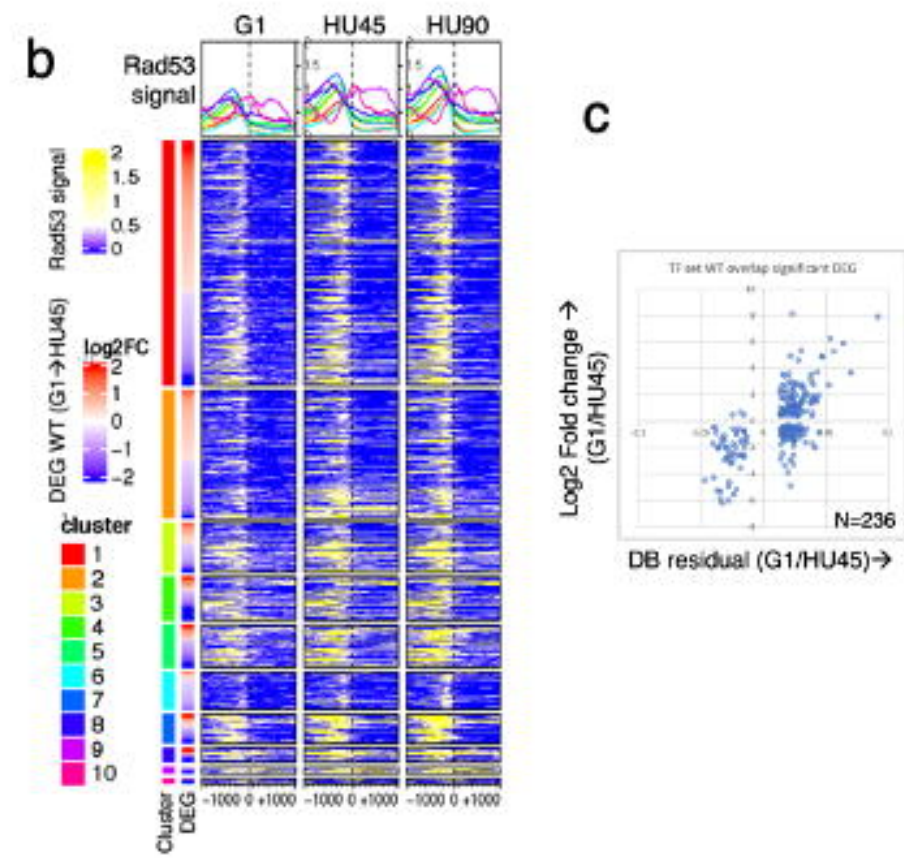

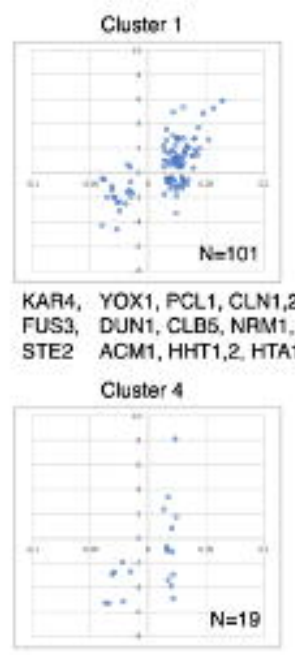

MFA2, AGA2, FUS2.

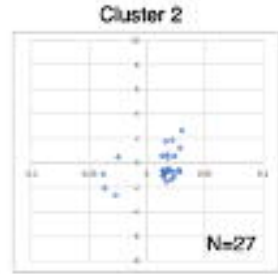

HHO1, TOS2, SKM1..

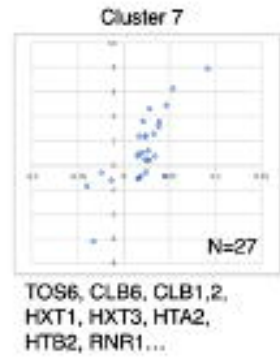




\section{Figure 6 - figure supplement 1}

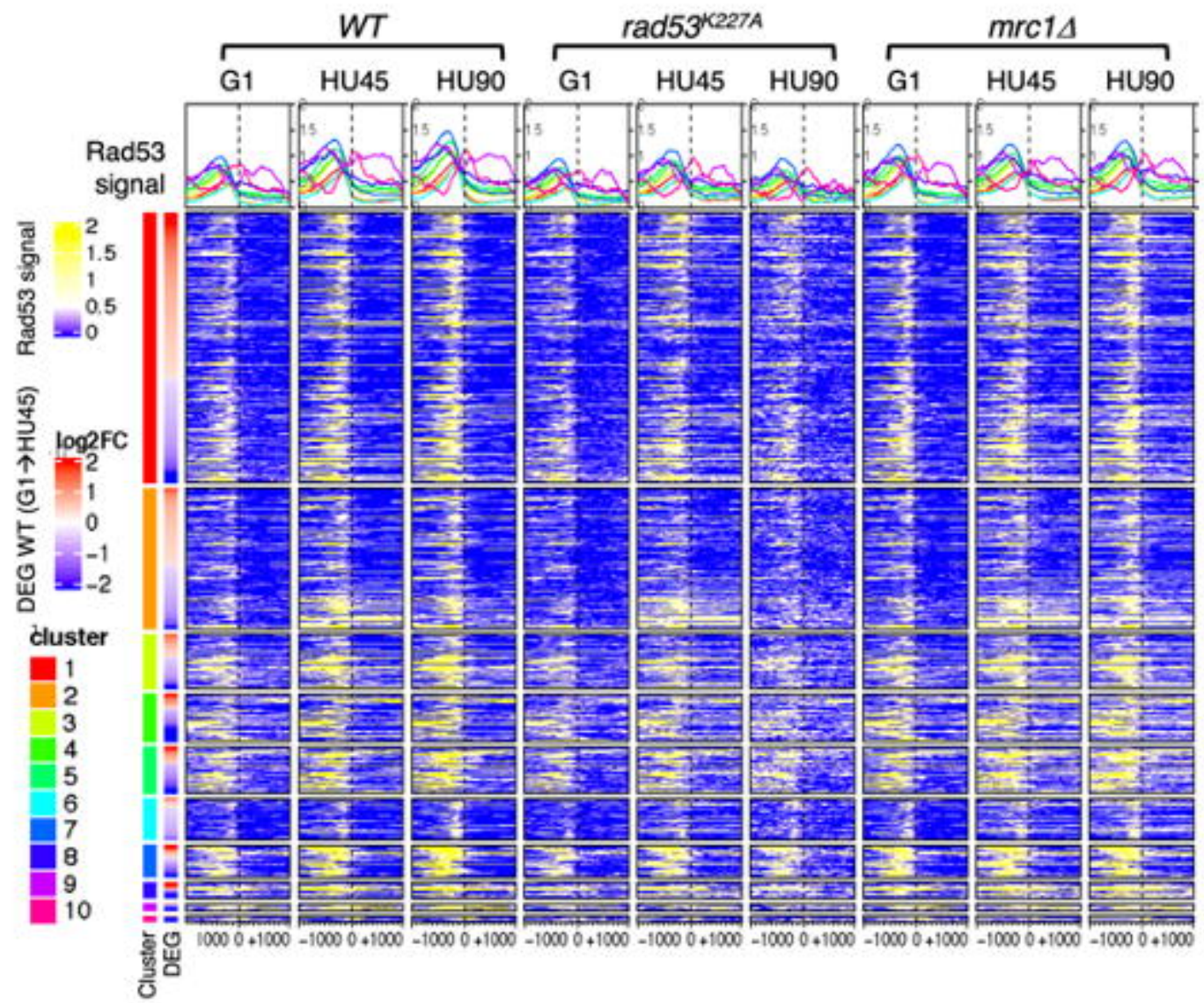




\section{Figure 7}

a

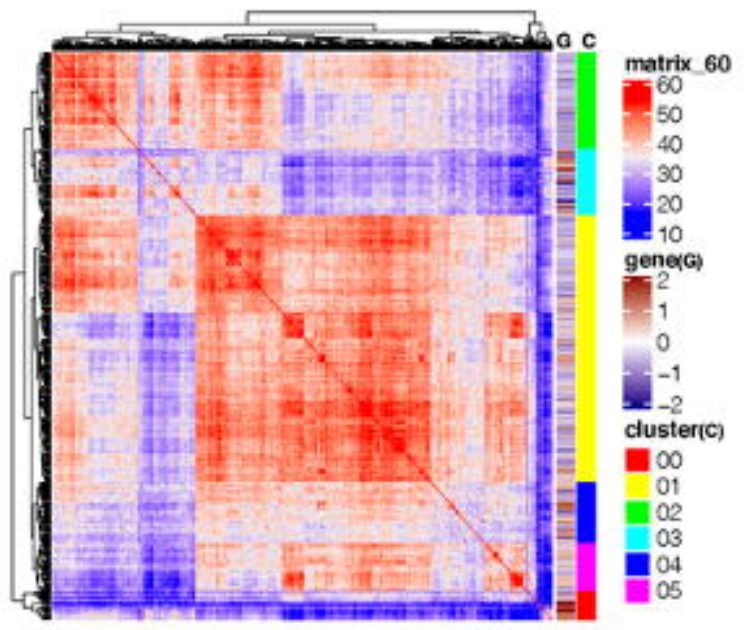

Summary of GO function DEG clusters for HU45 (mre1A vs WT)

1. (up) mitotic cell cycle, cytokinesis

1. (down) cell cycle checkpoint; ubiquitin-dependent protein catabolic process; proteolysis

\section{4. organic acid biosynthetic process}

5. chromatin assembly or disassembly (histone genes)

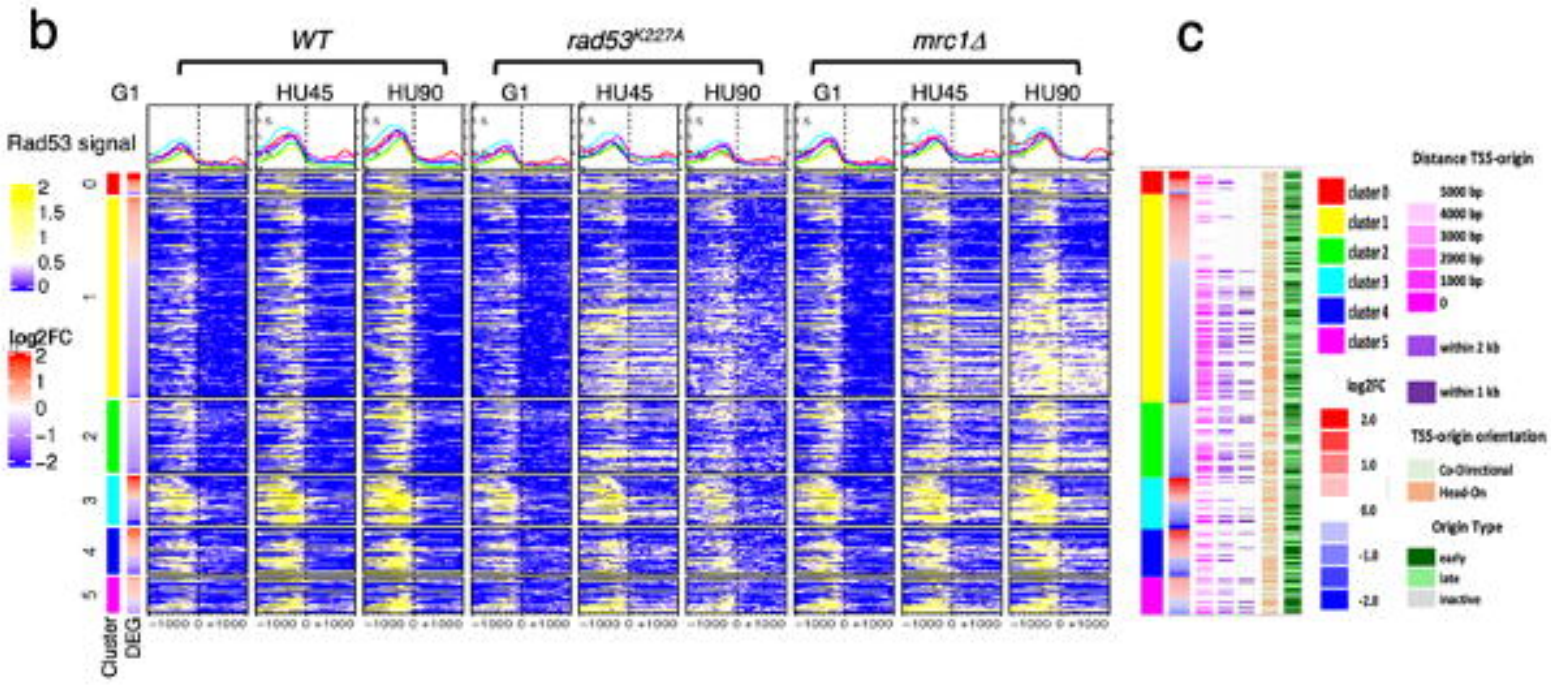

d
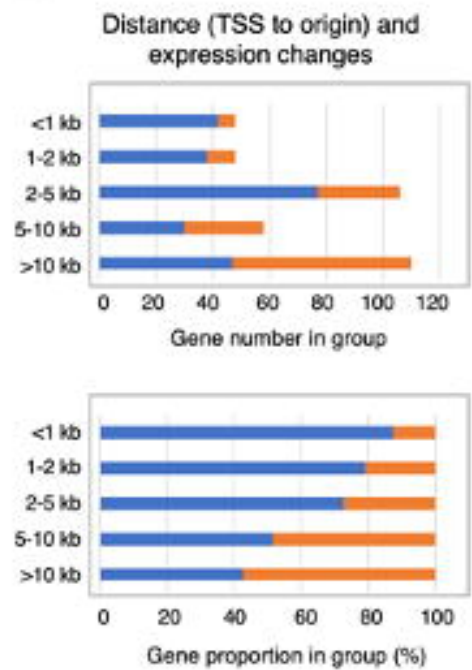

DEG down-fegulated

DEG up-regulated

Origin type within $5 \mathrm{~kb}$
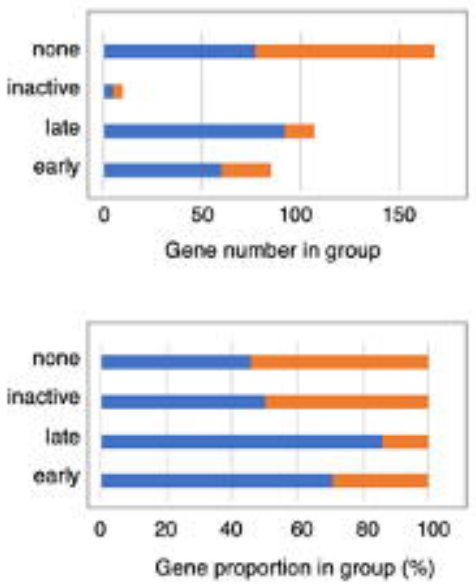

Origin-gene orientation and expression changes
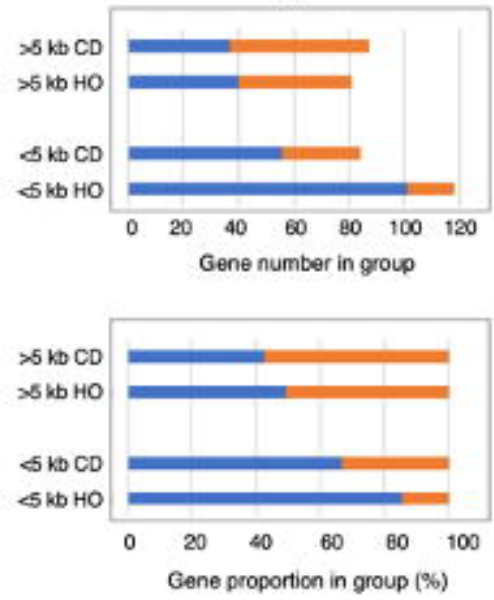


\section{Figure 7 - figure supplement 1}

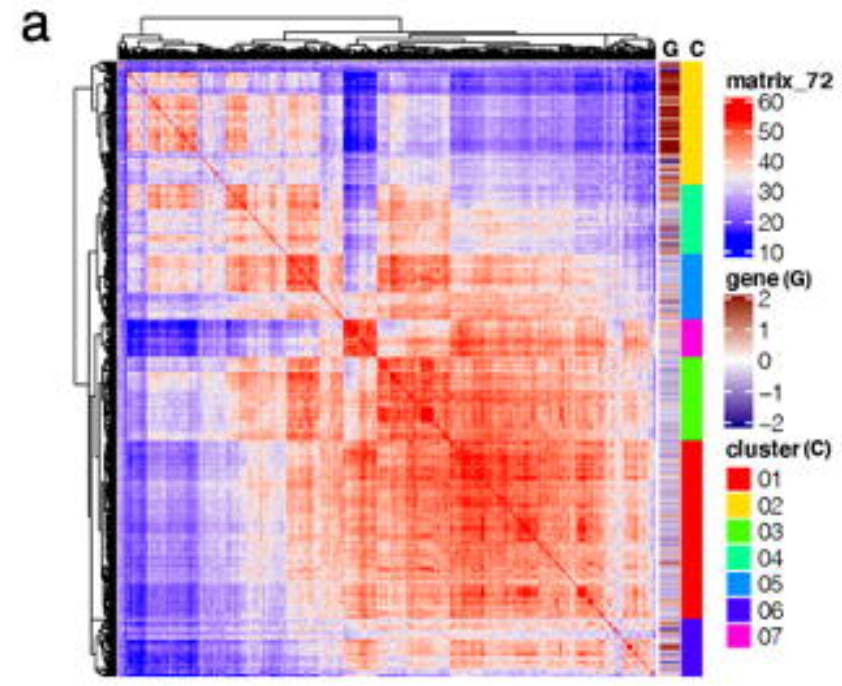

\section{Summary of GO function: \\ DEG clusters HU45 (rad53 ${ }^{\text {K227A }}$ vs WT)}

\begin{tabular}{|l|}
\hline $\begin{array}{l}\text { 1. cell cycle, replication, repair, } \\
\text { negative regulation of gene } \\
\text { expression }\end{array}$ \\
\hline $\begin{array}{l}\text { Mitosis, MT and spindle function, } \\
\text { cell division, cytokinesis }\end{array}$ \\
\hline
\end{tabular}

2. oxidation-reduction process, energy derivation

\section{3. proteolysis; actin cytoskeleton} organization and ER stress

6. nucleotide, oxidation-reduction process; all histone genes

7. ribosome biogenesis

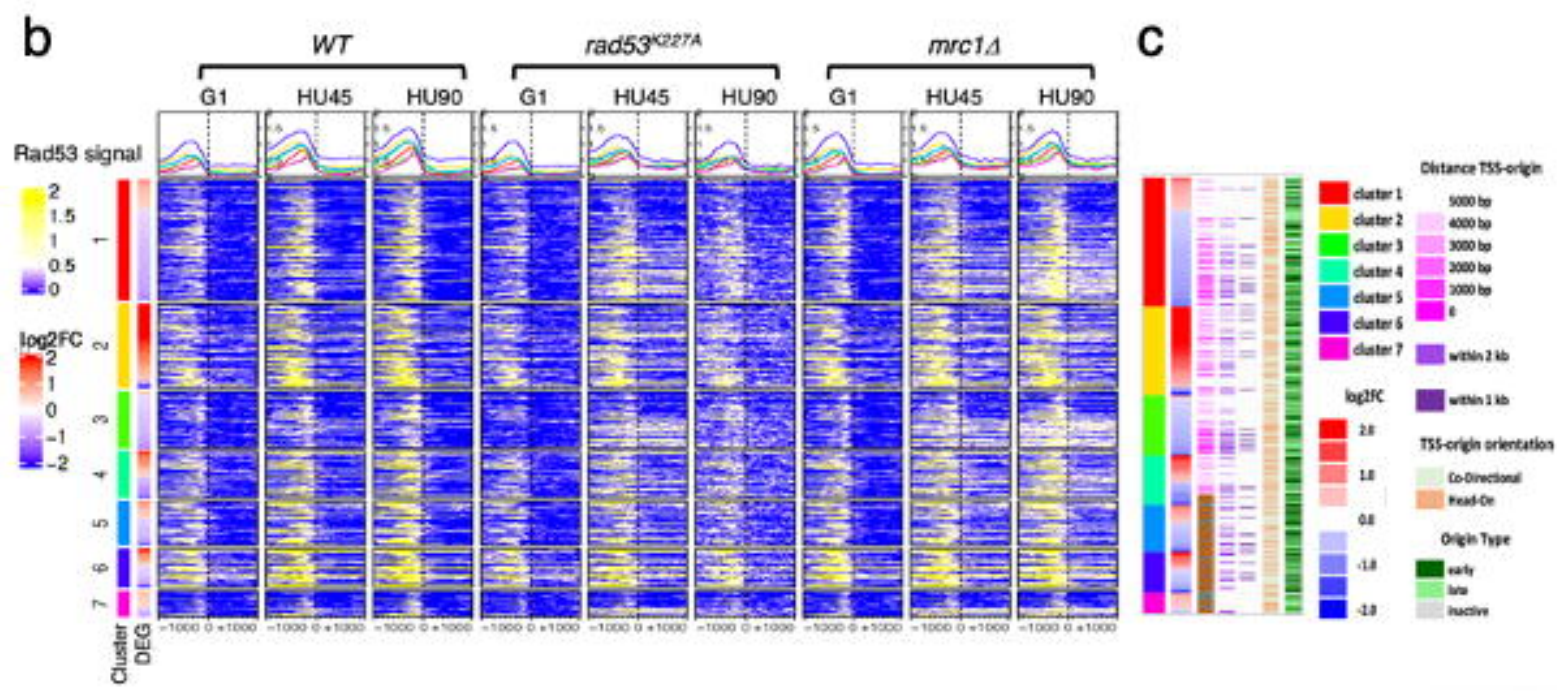


a Targets of:

SBF

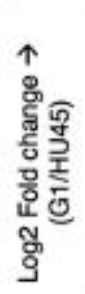

MBF

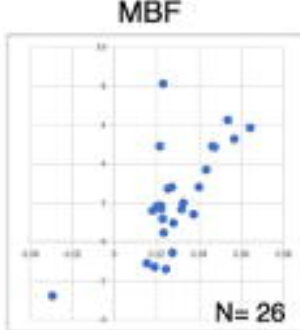

Ste 12

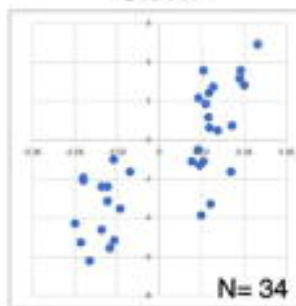

Msn4

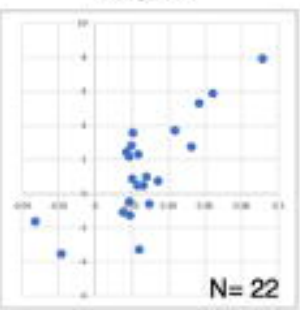

$\mathrm{N}=22$

DB residual (G1/HU45) $\rightarrow$

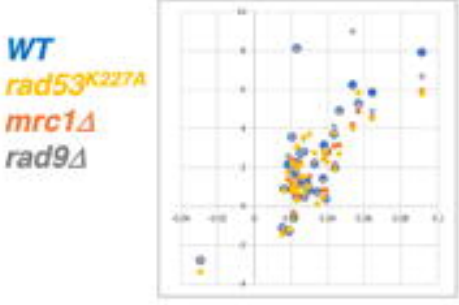

Enrichment:

12.91

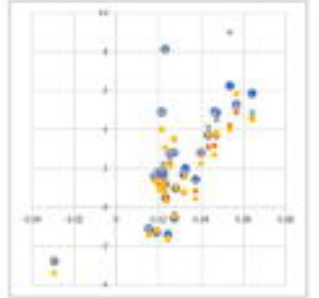

11.62

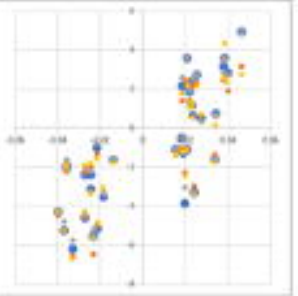

5.40

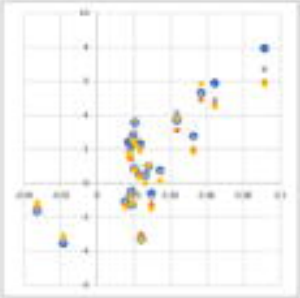

3.20 b

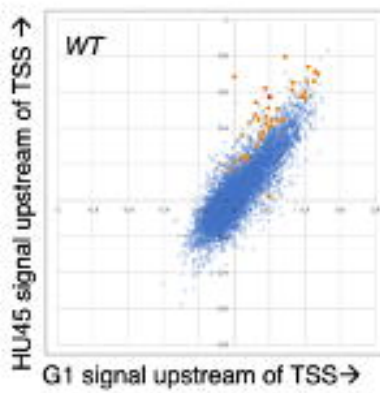

+4 SBF targets

- RNR1 ixris

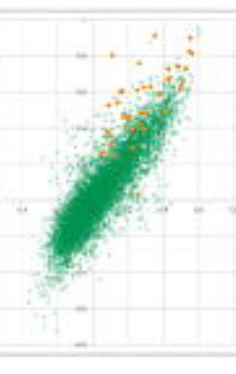

swi6

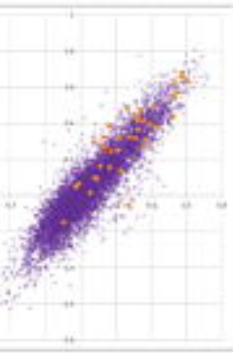

swi4 4
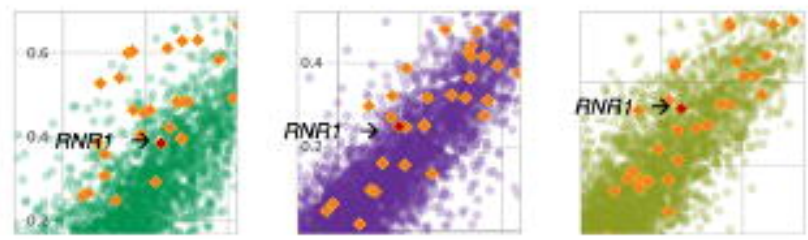

c

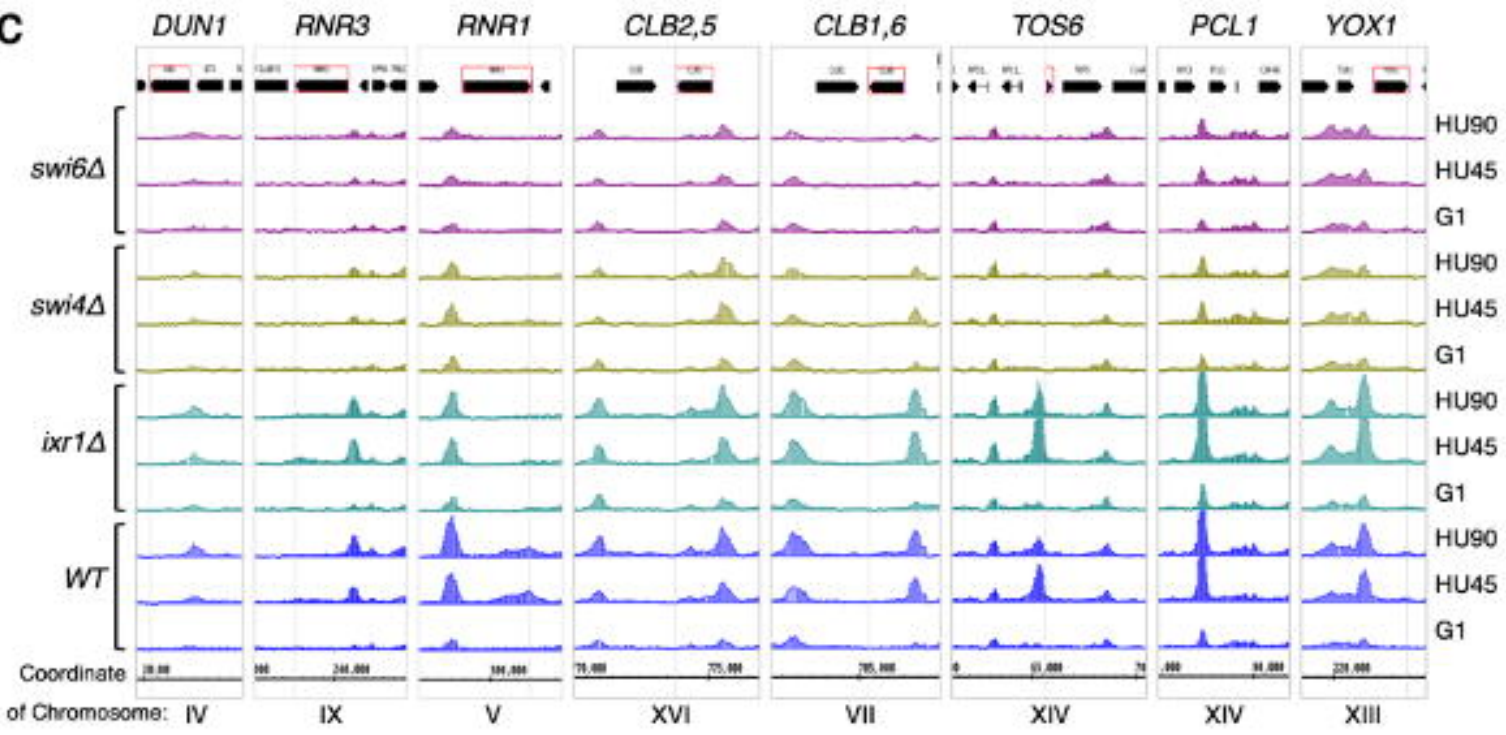


a

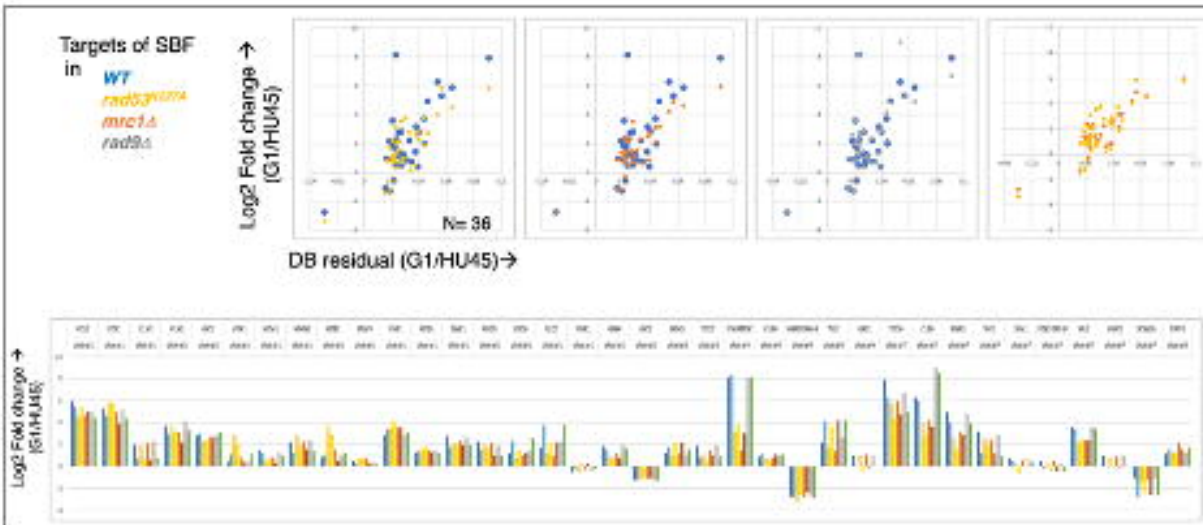

b

Targets of MBF

in $w T$ misota rodis
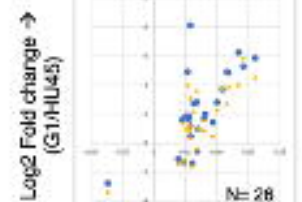

$\mathrm{DB}$ residual (G1/HU45) $\rightarrow$

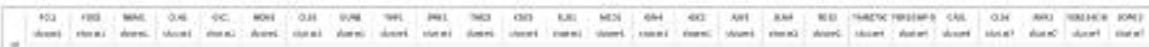

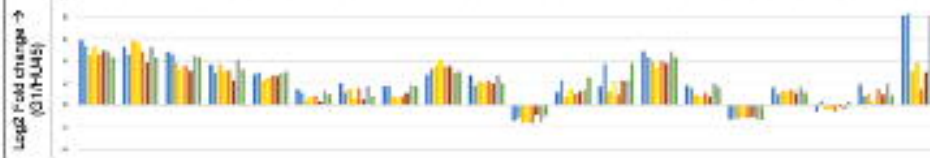

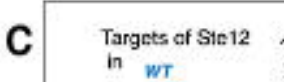

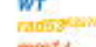
$\underset{m a t c t s}{\operatorname{math}}$
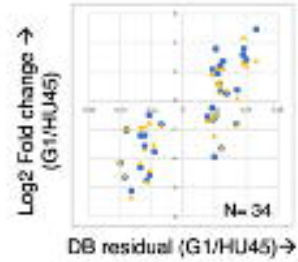

DB residual $\{$ G $1 /$ HU45) $\rightarrow$

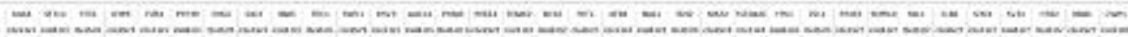

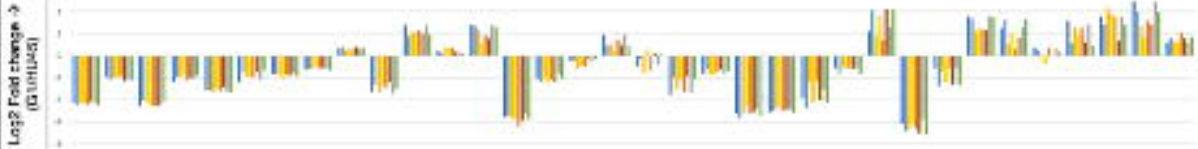

d Tergets of Man4
in wr
madsanamen
radis

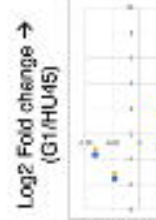
$\mathrm{DB}$ residual (G1HUL45) $\rightarrow$
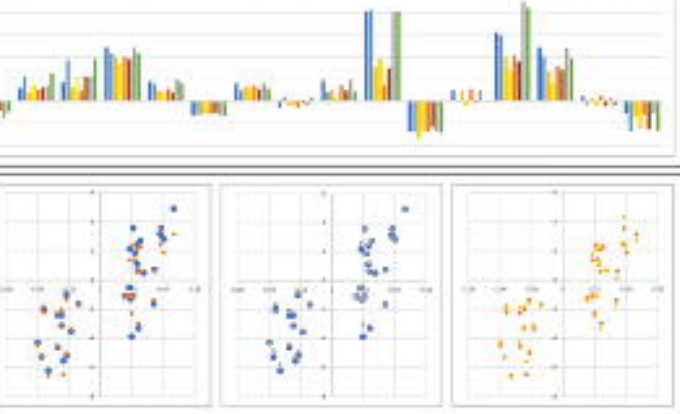

\section{Figure 8 - figure supplement 1}

Sample order in columns
WT $\operatorname{rad53} \times 2274$

mre1s

HU9O 